Article

\title{
Optimal Portfolio Selection in an Itô-Markov Additive Market
}

\author{
Zbigniew Palmowski ${ }^{1, * \mathbb{D}}$, Łukasz Stettner ${ }^{2,3}$ and Anna Sulima 4 \\ 1 Department of Applied Mathematics, Faculty of Pure and Applied Mathematics, Wrocław University of \\ Science and Technology, 50-370 Wrocław, Poland \\ 2 Institute of Mathematics, Polish Academy of Sciences, 00-656 Warsaw, Poland; stettner@impan.pl \\ 3 Vistula University, 02-787 Warsaw, Poland \\ 4 Faculty of Management, Computer Science and Finance, Wrocław University of Economics, 53-345 Wrocław, \\ Poland; anna.sulima@wp.pl \\ * Correspondence: zbigniew.palmowski@pwr.edu.pl or zbigniew.palmowski@gmail.com; \\ Tel.: +48-71-320-2012
}

Received: 8 December 2018; Accepted: 18 March 2019; Published: 25 March 2019

\begin{abstract}
We study a portfolio selection problem in a continuous-time Itô-Markov additive market with prices of financial assets described by Markov additive processes that combine Lévy processes and regime switching models. Thus, the model takes into account two sources of risk: the jump diffusion risk and the regime switching risk. For this reason, the market is incomplete. We complete the market by enlarging it with the use of a set of Markovian jump securities, Markovian power-jump securities and impulse regime switching securities. Moreover, we give conditions under which the market is asymptotic-arbitrage-free. We solve the portfolio selection problem in the Itô-Markov additive market for the power utility and the logarithmic utility.
\end{abstract}

Keywords: Markov additive processes; Markov regime switching market; Markovian jump securities; asymptotic arbitrage; complete market; optimal portfolio

\section{Introduction}

The portfolio selection problem is an important issue in financial mathematics. The problem is to invest an initial wealth in financial assets so as to maximize the expected utility of the terminal wealth. Markowitz (1952) for the first time used the quantitative methods for the optimal portfolio selection problem and proposed the mean-variance approach for portfolio optimization. Explicit solutions for the portfolio selection problem in continuous time were first given by Merton $(1971,1980)$.

Although Merton's approach produces significant theoretical results, it has some shortcomings coming from daily practice. The first is related to the assumption that the dynamics of a risky asset follows a geometric Brownian motion. Many investigations (e.g., Black et al. (1972) and Merton (1976)) have suggested that this market model cannot describe perfectly some empirical behaviors of financial markets, such as the asymmetry and heavy-tailedness of the distribution of returns of a time-varying volatility. To model this, the stock price driven by a Lévy process is a better choice. The portfolio selection problem on a Lévy market was considered by Niu (2008) and Corcuera et al. (2006).

The second important assumption in the original Black-Scholes-Merton model is that the coefficients are fixed. However, this assumption may not be satisfied over long time period, where structural changes in macroeconomic conditions may occur several times. Therefore, Markov modulated models (otherwise called regime switching) were proposed instead. In such models, the set of model parameters change in time according to a Markov chain, the transitions of which correspond to changes in the state of the economy. Hence, regime switching models describe perfectly structural macroeconomic changes and various business cycles (Zhang 2001). Hamilton (1989) pioneered 
econometric applications of regime switching models. These models have many applications in finance (Buffington and Elliott 2002; Di Masi et al. 1994; Elliott et al. 2001, 2003, 2005; Goldfeld and Quandt 1973; Guo 2001; Naik 1993; Tong 1978).

There is a growing literature dealing with portfolio optimization problems in markets with non-constant coefficients. Most of these papers assume that the external process is a diffusion process itself, like in the established volatility model of Heston (1993) or in the Ho-Lee and the Vasicek model of Korn and Kraft (2001). Bäuerle and Rieder (2004) and Rieder and Bäuerle (2005) studied the portfolio optimization problem with an observable and an unobservable Markov-modulated drift, respectively. This problem under stochastic volatility was considered by Pham and Quenez (2001) and Fleming and Hernández-Hernández (2003). In contrast to diffusion volatility, Markov chain volatility has the advantage that many portfolio problems have explicit solution. Moreover, a diffusion process can be approximated arbitrarily closely by a continuous-time Markov chain (Kushner and Dupuis 1992).

Portfolio optimization problems have also been studied in financial markets with regime switching. One of the first papers was by Zariphopoulou (1992), who maximized the utility of consumption under proportional transaction costs in a market where stock returns are determined by a continuous-time Markov chain, and established a viscosity property of the value function. The results of Zariphopoulou were extended by many authors, among them Bäuerle and Rieder $(2004,2007)$, Fontana et al. (2015), Framstad et al. (2004), Zhang and Yin (2004) and Stockbridge (2002). To solve the problem of maximizing the investor's expected utility of terminal wealth, some authors used numerical methods (Sass and Haussmann 2004; Nagai and Runggaldier 2008; Shen et al. 2012; Fu et al. 2014). Zhang et al. (2010) solved the portfolio selection problem without transition cost in a continuous-time Markovian regime switching Black-Scholes-Merton market. They obtained closed-form solutions for the optimal portfolio strategies when utility function is logarithmic or power-type. Similar results for a Black-Scholes market with regime switching were obtained by Liu (2014), Guo et al. (2005) and Sotomayor and Cadenillas (2013). A discrete time set up was also considered by Yin and Zhou (2004). For the mean-variance portfolio selection problem of this type, we refer to Zhou and Yin (2003). Regime switching was also analyzed by $\mathrm{Tu}$ (2010) in a Bayesian setting with model uncertainty and parameter uncertainty. He showed that the economic cost of ignoring regime switching can exceed two percent per year. Bae et al. (2014) constructed a program to optimize portfolios in the above mentioned framework and proved that adding Markov modulation improves risk management. Finally, further applications include large investor models Busch et al. (2013) and optimal productmanagement Korn et al. (2017).

In this paper, we consider a market with the prices of financial assets described by Itô-Markov additive processes, which combine Lévy processes and regime switching models. Such a process evolves as an Itô-Lévy process between changes of states of a Markov chain, that is, its parameters depend on the current state of the Markov chain. In addition, a transition of the Markov chain from state $i$ to state $j$ triggers an additional jump. Itô-Markov additive processes are classical in modeling queues, insurance risks, inventories, data communication, finance, environmental problems and in many other applications (Asmussen 2003; Prabhu 1998, chp. 7, and references therein).

The goal of this paper is to construct a general approach of building the optimal portfolio taking into account the asset jumps and possibility of changing environment by considering asset prices modeled by Itô-Markov additive processes. In particular, we assume that the interest rate and the volatility of the financial assets depend on a continuous-time finite-state Markov chain. Thus, our model takes into account two sources of risk: the jump diffusion risk and the regime switching risk. The jump diffusion risk refers to the source of risk due to fluctuations of market prices modeled by a Poisson random measure, while the regime switching risk refers to the source of risk due to transitions of economic conditions.

Due to the presence of these sources of risk, our market model is incomplete. In this paper, we show how to complete the Itô-Markov additive market model by adding Markovian jump securities, Markovian power-jump securities and impulse regime switching securities. Using these securities, all contingent claims can be replicated by a self-financing portfolio. The main idea 
of completing a Markovian regime switching market is inspired by Corcuera et al. (2005, 2006), Guo (2001), Karatzas et al. (1991), Niu (2008) and Zhang et al. (2012). However, adding the possibility of jumps of underlying markets when Markov chain changes its state produces more complex analysis that one presented in Corcuera et al. $(2005,2006)$.Moreover, we give conditions for the market to be asymptotic-arbitrage-free, namely, we find a martingale measure under which all the discounted price processes are martingales.

In this paper, we also consider the problem of identifying the optimal strategy that maximizes the expected value of the utility function of the wealth process at the end of some fixed period. The analysis is conducted for the logarithmic and power utility functions. To solve the main problem of determining the optimal portfolio we do not use dynamic programming but the direct differentiation approach.

This paper is organized as follows. In Section 2, we present the dynamics of the price process in an Itô-Markov additive market. In Section 3, we enlarge this market by Markovian jump securities, Markovian power-jump securities and impulse regime switching securities. In Sections 4 and 5, we show that the enlarged market is asymptotic-arbitrage-free and complete. In Section 6, we state the portfolio optimization problem and solve it for the power utility with risk aversion and the logarithmic utility function. Moreover, Section 7 gives a relationship between finite and infinite markets.

\section{Market Model}

Let $(\Omega, \mathcal{F}, \mathbb{P})$ be a complete probability space and let $\mathbb{T}:=[0, T]$, for fixed $0<T<\infty$, represent the maturity time for all economic activities. On this probability space, we consider the observable and continuous-time Markov chain $J:=\{J(t): t \in \mathbb{T}\}$ with a finite state space. The role of the Markov chain is to ensure that the parameters change according to the market environment and the different states of the Markov chain represent the different states of the economy. For simplicity, we follow the notation of Elliott et al. (1994) and we identify the state space with the standard basis $E:=\left\{\mathbf{e}_{1}, \ldots, \mathbf{e}_{N}\right\}$. Here, $\mathbf{e}_{i} \in \mathbb{R}^{N}$ and the $j$ th component of $\mathbf{e}_{i}$ is the Kronecker delta $\delta_{i j}$ for each $i, j=1, \ldots, N$. Moreover, the Markov chain $J$ is characterized by an intensity matrix $\left[\lambda_{i j}\right]_{i, j=1}^{N}$. The element $\lambda_{i j}$ is the transition intensity of the Markov chain $J$ jumping from state $\mathbf{e}_{i}$ to state $\mathbf{e}_{j}$. We assume $\lambda_{i j}>0$ for $i \neq j$. Note that $\sum_{j=1}^{N} \lambda_{i j}=0$, thus $\lambda_{i i}<0$.

\subsection{Risk-Free Asset}

Now, we describe the dynamic of the price process of risk-free asset $B$ as follows:

$$
\mathrm{d} B(t)=r(t) B(t) \mathrm{d} t, \quad B(0)=1 .
$$

Here, $r$ is the interest rate of $B$ and it is modulated by Markov chain $J$

$$
r(t):=\langle\mathbf{r}, J(t)\rangle=\sum_{i=1}^{N} r_{i}\left\langle\mathbf{e}_{i}, J(t)\right\rangle,
$$

where $\mathbf{r}=\left(r_{1}, \ldots, r_{N}\right)^{\prime} \in \mathbb{R}_{+}^{N}$ and $\langle\cdot, \cdot\rangle$ is a scalar product in $\mathbb{R}^{N}$. The value $r_{i}$ represents the value of the interest rate when the Markov chain is in the state space $\mathbf{e}_{i}$.

\subsection{Risky Asset}

We consider the market with risky asset modeled by (non-anticipative) Itô-Markov additive processes.

A process $(J, X)=\{(J(t), X(t)): t \in \mathbb{T}\}$ on the state space $\left\{\mathbf{e}_{1}, \ldots, \mathbf{e}_{N}\right\} \times \mathbb{R}$ is a Markov additive process (MAP) if $(J, X)$ is a Markov process and the conditional distribution of $(J(s+t), X(s+t)-$ $X(s))$ for $s, t \in \mathbb{T}$, given $(J(s), X(s))$, depends only on $J(s)$ (Çinlar 1972a, 1972b). Every MAP has a very special structure. It is usually said that $X$ is the additive component and $J$ is the background 
process representing the environment. Moreover, the process $X$ evolves as a Itô-Lévy process while $J(t)=\mathbf{e}_{j}$, that is, $X(t)=X^{j}(t)$ for some Itô-Lévy process $X^{j}(t)$ with parameters depending on $\mathbf{e}_{j}$ if $J(t)=\mathbf{e}_{j}$. In addition, transition of $J$ from $\mathbf{e}_{i}$ to $\mathbf{e}_{j}$ triggers a jump of $X$ distributed as $U_{n}^{(i)}$.

Following Asmussen and Kella (2000), we can decompose the process $X$ as follows:

$$
X(t)=\bar{X}(t)+\overline{\bar{X}}(t)
$$

where

$$
\overline{\bar{X}}(t):=\sum_{i=1}^{N} \Psi_{i}(t)
$$

for

$$
\Psi_{i}(t):=\sum_{n \geq 1} U_{n}^{(i)} \mathbf{1}_{\left\{J\left(T_{n}-\right)=\mathbf{e}_{i}, T_{n} \leq t\right\}}
$$

and for the jump epochs $\left\{T_{n}\right\}$ of $J$. Here, $U_{n}^{(i)}(n \geq 1,1 \leq i \leq N)$ are independent random variables, which are also independent of $\bar{X}$ such that, for every fixed $i$, the random variables $U_{n}^{(i)}$ are identically distributed. Note that we can express the process $\Psi_{i}$ as follows:

$$
\Psi_{i}(t)=\int_{0}^{t} \int_{\mathbb{R}} x \Pi_{U}^{i}(\mathrm{~d} s, \mathrm{~d} x)
$$

for the point measure

$$
\Pi_{U}^{i}([0, t], \mathrm{d} x):=\sum_{n \geq 1} \mathbf{1}_{\left\{U_{n}^{(i)} \in \mathrm{d} x\right\}} \mathbf{1}_{\left\{J\left(T_{n}\right)=\mathbf{e}_{i}, T_{n} \leq t\right\}}, \quad i=1, \ldots, N .
$$

Moreover, we define the compensated point measure $\bar{\Pi}_{U}^{i}(\mathrm{~d} t, \mathrm{~d} x):=\Pi_{U}^{i}(\mathrm{~d} t, \mathrm{~d} x)-\lambda_{i}(t) \eta_{i}(\mathrm{~d} x) \mathrm{d} t$ for $\lambda_{j}(t):=\sum_{i \neq j} \mathbf{1}_{\left\{J(t-)=\mathbf{e}_{i}\right\}} \lambda_{i j}$ and $\eta_{i}(\mathrm{~d} x)=\mathbb{P}\left(U_{n}^{(i)} \in \mathrm{d} x\right)$.

Remark 1. One can consider jumps $U^{(i j)}$ with distribution depending also on the state $\boldsymbol{e}_{j}$ the Markov chain is jumping to by extending the state space to the pairs $\left(\boldsymbol{e}_{i}, \boldsymbol{e}_{j}\right)$ (see Gautam et al. $(1999, \mathrm{Thm}$. 5) for details).

The first component in the definition in Equation (2) is an Itô-Lévy process and it has the following decomposition (Øksendal and Sulem 2004, p. 5):

$$
\bar{X}(t):=\bar{X}(0)+\int_{0}^{t} \mu_{0}(s) \mathrm{d} s+\int_{0}^{t} \sigma_{0}(s) \mathrm{d} W(s)+\int_{0}^{t} \int_{\mathbb{R}} \gamma(s-, x) \bar{\Pi}(\mathrm{d} s, \mathrm{~d} x),
$$

where $W$ denotes the standard Brownian motion independent of $J$ and $\Pi(\mathrm{d} t, \mathrm{~d} x):=\Pi(\mathrm{d} t, \mathrm{~d} x)-$ $v(\mathrm{~d} x) \mathrm{d} t$ is the compensated Poisson random measure, which is independent of $J$ and $W$. Furthermore, we define

$$
\begin{gathered}
\mu_{0}(t):=\left\langle\boldsymbol{\mu}_{0}, J(t)\right\rangle=\sum_{i=1}^{N} \mu_{0}^{i}\left\langle\mathbf{e}_{i}, J(t)\right\rangle, \\
\sigma_{0}(t):=\left\langle\sigma_{0}, J(t)\right\rangle=\sum_{i=1}^{N} \sigma_{0}^{i}\left\langle\mathbf{e}_{i}, J(t)\right\rangle, \\
\gamma(t, x):=\langle\gamma(x), J(t)\rangle=\sum_{i=1}^{N} \gamma_{i}(x)\left\langle\mathbf{e}_{i}, J(t)\right\rangle
\end{gathered}
$$

for some vectors $\mu_{0}:=\left(\mu_{0}^{1}, \ldots, \mu_{0}^{N}\right)^{\prime} \in \mathbb{R}^{N}, \sigma_{0}:=\left(\sigma_{0}^{1}, \ldots, \sigma_{0}^{N}\right)^{\prime} \in \mathbb{R}_{+}^{N}$ with $\sigma_{0}^{j}>0$ and the vector-valued measurable function $\gamma(x):=\left(\gamma_{1}(x), \ldots, \gamma_{N}(x)\right)$. The measure $v$ is the so-called jump-measure identifying the distribution of the sizes of the jumps of the Poisson measure $\Pi$. 
The components $\bar{X}$ and $\overline{\bar{X}}$ in Equation (2) are conditionally, on the state of the Markov chain $J$, independent.

Additionally, we suppose that the Lévy measure satisfies, for some $\varepsilon>0$ and $\varrho>0$,

$$
\int_{(-\varepsilon, \mathcal{E})^{c}} \exp (\varrho|\gamma(s-, x)|) v(\mathrm{~d} x)<\infty, \quad \int_{(-\varepsilon, \mathcal{E})^{c}} \exp (\varrho x) \mathbb{P}\left(U^{(i)} \in \mathrm{d} x\right)<\infty,
$$

for $i=1, \ldots, N$. This implies that

$$
\int_{\mathbb{R}}|\gamma(s-, x)|^{k} v(\mathrm{~d} x)<\infty, \quad \mathbb{E}\left(U^{(i)}\right)^{k}<\infty, \quad i=1, \ldots, N, \quad k \geq 2,
$$

and that the characteristic function $\mathbb{E}[\exp (k i X)]$ is analytic in a neighborhood of 0 . Moreover, $X$ has moments of all orders and the polynomials are dense in $L^{2}(\mathbb{R}, \mathrm{d} \varphi(t, x))$, where $\varphi(t, x):=\mathbb{P}(X(t) \leq x)$. Itô-Markov additive processes are a natural generalization of Itô-Lévy processes and thus of Lévy processes. Moreover, the structure in Equation (2) explains the used name and can be seen as Markov-modulated Itô-Lévy process. Indeed, if $\gamma(s, x)=x$, then $X$ is a Markov additive process. If additionally $N=1$, then $X$ is a Lévy process. If $U^{(i)} \equiv 0$ and $N>1$ then $X$ is a Markov modulated Lévy process (Pacheco et al. 2009). If there are no jumps, that is, $\bar{\Pi}(\mathrm{d} s, \mathrm{~d} x)=0$, we have a Markov modulated Brownian motion.

We assume the evolution of the price process of the risky asset $S_{0}$ is governed by the Itô-Markov additive process as follows:

$$
\left\{\begin{array}{l}
\mathrm{d} S_{0}(t)=S_{0}(t-)\left[\mu_{0}(t) \mathrm{d} t+\sigma_{0}(t) \mathrm{d} W(t)+\int_{\mathbb{R}} \gamma(t-, x) \bar{\Pi}(\mathrm{d} t, \mathrm{~d} x)+\sum_{i=1}^{N} \int_{\mathbb{R}} x \bar{\Pi}_{U}^{i}(\mathrm{~d} t, \mathrm{~d} x)\right], \\
S_{0}(0)=s_{0}>0 .
\end{array}\right.
$$

To ensure that $S_{0}(t)$ is non-negative, we additionally assume that

$$
\Delta \int_{\mathbb{R}} \gamma(t-, x) \bar{\Pi}(\mathrm{d} t, \mathrm{~d} x)>-1, \quad \Delta \int_{\mathbb{R}} x \bar{\Pi}_{U}^{i}(\mathrm{~d} t, \mathrm{~d} x)>-1, \quad i=1, \ldots N,
$$

where $\Delta Z_{t}=Z_{t}-Z_{t-}$ is a jump process related with a processes $Z$. The last assumption is equivalent to

$$
\Delta \Psi_{i}(t)>-1
$$

and it is satisfied when all $U^{i}(i=1, \ldots, N)$ have support included in $(-1,+\infty)$. We interpret the coefficient $\mu_{0}$ defined in Equation (6) as the appreciation rate and $\sigma_{0}$ defined in Equation (7) as the volatility of the risky asset for each $i=1, \ldots, N$. Similarly, $\mu_{0}^{i}$ and $\sigma_{0}^{i}$ represent the appreciation rate and the volatility of the risky asset, respectively, when the Markov chain is in state $\mathbf{e}_{i}$. We assume that

$$
\mu_{0}^{i}>r_{i}, \quad i=1, \ldots, N
$$

Otherwise, stocks would be just a bad investment.

\section{Enlarging the Itô-Markov Additive Market}

Now, we enlarge the primary market by some financial assets: Markovian jump securities, Markovian power-jump securities and impulse regime switching securities in order to complete the market.

From now, we work with the following filtration on $(\Omega, \mathcal{F}, \mathbb{P})$ :

$$
\mathcal{F}_{t}:=\mathcal{G}_{t} \vee \mathcal{N},
$$


where $\mathcal{N}$ are the $\mathbb{P}$-null sets of $\mathcal{F}$ and

$$
\mathcal{G}_{t}:=\sigma\left\{J(s), W(s), \Gamma(s), \Pi_{U}^{1}([0, s], \mathrm{d} x), \ldots, \Pi_{U}^{N}([0, s], \mathrm{d} x) ; s \leq t\right\}
$$

for

$$
\Gamma(t):=\int_{0}^{t} \int_{\mathbb{R}} \gamma(s-, x) \Pi(\mathrm{d} s, \mathrm{~d} x) .
$$

Note that the filtration $\left\{\mathcal{F}_{t}\right\}_{t \geq 0}$ is right-continuous (Karatzas and Shreve 1998, Prop. 7.7 and also Protter 2005, Thm. 31). By the same arguments as in the proof of Thm. 3.3. of Liao (2004), the filtration $\left\{\mathcal{G}_{t}\right\}_{t \geq 0}$ can be represented as

$$
\sigma\left\{J(s), \bar{X}(s), \Pi_{U}^{1}([0, s], \mathrm{d} x), \ldots, \Pi_{U}^{N}([0, s], \mathrm{d} x) ; s \leq t\right\} .
$$

\subsection{Markovian Jump Securities}

Let $T_{n}(n=1,2, \ldots)$ denote the jump epochs of the chain $J$, where $0<T_{1}<T_{2}<\ldots$ We observe that the Markov chain $J$ can be represented in terms of a marked point process $\Phi_{j}$ defined by

$$
\Phi_{j}(t):=\Phi\left([0, t] \times \mathbf{e}_{j}\right)=\sum_{n \geq 1} \mathbf{1}_{\left\{J\left(T_{n}\right)=\mathbf{e}_{j}, T_{n} \leq t\right\}}, j=1, \ldots, N .
$$

Note that the process $\Phi_{j}$ describes the number of jumps into state $\mathbf{e}_{j}$ up to time $t$. Let $\phi_{j}$ be the dual predictable projection of $\Phi_{j}$ (sometimes called the compensator). That is, the process

$$
\bar{\Phi}_{j}(t):=\Phi_{j}(t)-\phi_{j}(t), j=1, \ldots, N,
$$

is an $\left\{\mathcal{F}_{t}\right\}$-martingale and it is called the $j$ th Markovian jump martingale. Note that $\phi_{j}$ is unique and

$$
\phi_{j}(t):=\int_{0}^{t} \lambda_{j}(s) \mathrm{d} s
$$

for

$$
\lambda_{j}(t):=\sum_{i \neq j} \mathbf{1}_{\left\{J(t-)=\mathbf{e}_{i}\right\}} \lambda_{i j}
$$

see Zhang et al. (2012, p. 290).

Now, we consider geometric Markovian jump securities $S_{j}($ for $j=1, \ldots, N$ ) with evolution of prices described by marked point local martingales as follows:

$$
\left\{\begin{array}{l}
\mathrm{d} S_{j}(t)=S_{j}(t-)\left[\mu_{j}(t) \mathrm{d} t+\sigma_{j}(t-) \mathrm{d} \bar{\Phi}_{j}(t)\right] \\
S_{j}(0)>0
\end{array}\right.
$$

where the appreciation rate $\mu_{j}$ and the volatility $\sigma_{j}$ are given by

$$
\begin{aligned}
& \mu_{j}(t):=\left\langle\boldsymbol{\mu}_{j}, J(t)\right\rangle=\sum_{i=1}^{N} \mu_{j}^{i}\left\langle\mathbf{e}_{i}, J(t)\right\rangle, \\
& \sigma_{j}(t):=\left\langle\sigma_{j}, J(t)\right\rangle=\sum_{i=1}^{N} \sigma_{j}^{i}\left\langle\mathbf{e}_{i} J(t)\right\rangle
\end{aligned}
$$

with $\mu_{j}:=\left(\mu_{j}^{1}, \ldots, \mu_{j}^{N}\right)^{\prime} \in \mathbb{R}^{N}$ and $\sigma_{j}:=\left(\sigma_{j}^{1}, \ldots, \sigma_{j}^{N}\right)^{\prime} \in \mathbb{R}_{+}^{N}$. 


\subsection{Markovian Power-Jump Securities}

Following Corcuera et al. (2005), we introduce the power-jump processes

$$
X^{(k)}(t):=\sum_{0<s \leq t}(\Delta \bar{X}(s))^{k}, \quad k \geq 2,
$$

where $\Delta \bar{X}(s)=\bar{X}(s)-\bar{X}(s-)$. We set $X^{(1)}(t)=\bar{X}(t)$. The process $X^{(k)}$ is also an Itô-Lévy process with the same jump times as the original process $\bar{X}$ but with their sizes being the $k$ th powers of the jump sizes of $\bar{X}$. From Protter (2005, p. 29), we have

$$
\mathbb{E}\left[X^{(k)}(t) \mid \mathcal{J}_{t}\right]=\mathbb{E}\left(\sum_{0<s \leq t}(\Delta \bar{X}(s))^{k} \mid \mathcal{J}_{t}\right)=\int_{0}^{t} \int_{\mathbb{R}} \gamma^{k}(s-, x) v(\mathrm{~d} x) \mathrm{d} s<\infty, \mathbb{P}-\text { a.e. } \quad k \geq 2,
$$

for $\mathcal{J}_{t}:=\sigma\{J(s): s \leq t\}$. Hence, the processes

$$
\bar{X}^{(k)}(t):=X^{(k)}(t)-\int_{0}^{t} \int_{\mathbb{R}} \gamma^{k}(s-, x) v(\mathrm{~d} x) \mathrm{d} s, \quad k \geq 2,
$$

are $\left\{\mathcal{F}_{t}\right\}$-martingales (called Teugels martingales of order $k$; see Schoutens (1999) for details).

We additionally enlarge the market with a series of Markovian $k$ th-power-jump assets $S^{(k)}$ (for $k \geq 2$ ). The price process of $S^{(k)}$ is described by the stochastic differential equation

$$
\left\{\begin{array}{l}
\mathrm{d} S^{(k)}(t)=S^{(k)}(t-)\left[\mu^{(k)}(t) \mathrm{d} t+\sigma^{(k)}(t-) \mathrm{d} \bar{X}^{(k)}(t)\right], \\
S^{(k)}(0)>0,
\end{array}\right.
$$

where the coefficients are determined by the Markov chain J, namely:

$$
\mu^{(k)}(t):=\left\langle\boldsymbol{\mu}^{(k)}, J(t)\right\rangle=\sum_{j=1}^{N} \mu_{j}^{(k)}\left\langle\mathbf{e}_{j}, J(t)\right\rangle \quad \text { and } \quad \sigma^{(k)}(t):=\left\langle\sigma^{(k)}, J(t)\right\rangle=\sum_{j=1}^{N} \sigma_{j}^{(k)}\left\langle\mathbf{e}_{j}, J(t)\right\rangle
$$

for $\boldsymbol{\mu}^{(k)}:=\left(\mu_{1}^{(k)}, \ldots, \mu_{N}^{(k)}\right)^{\prime} \in \mathbb{R}^{N}$ and $\sigma^{(k)}:=\left(\sigma_{1}^{(k)}, \ldots, \sigma_{N}^{(k)}\right)^{\prime} \in \mathbb{R}_{+}^{N}$. The positivity of these asset prices follow from assumption in Equation (11).

\subsection{Impulse Regime Switching Securities}

We will also need power martingales related to the second component of $X$ given in Equation (2), namely to $\overline{\bar{X}}$ or to $\Psi_{i}$, defined in Equation (3). For $l \geq 1$ and $i=1, \ldots, N$ we define

$$
\Psi_{i}^{(l)}(t):=\sum_{n \geq 1}\left(U_{n}^{(i)}\right)^{l} \mathbf{1}_{\left\{J\left(T_{n}\right)=\mathbf{e}_{i}, T_{n} \leq t\right\}}=\int_{0}^{t} \int_{\mathbb{R}} x^{l} \Pi_{U}^{i}(\mathrm{~d} s, \mathrm{~d} x)
$$

for $\Pi_{U}^{i}$ given by Equation (4). The compensated version of $\Psi_{i}^{(l)}$ is called an impulse regime switching martingale if

$$
\bar{\Psi}_{i}^{(l)}(t):=\Psi_{i}^{(l)}(t)-\mathbb{E}\left(U_{n}^{(i)}\right)^{l} \phi_{i}(t)=\int_{0}^{t} \int_{\mathbb{R}} x^{l} \bar{\Pi}_{U}^{i}(\mathrm{~d} s, \mathrm{~d} x),
$$

where $\bar{\Pi}_{U}^{i}(\mathrm{~d} t, \mathrm{~d} x)=\Pi_{U}^{i}(\mathrm{~d} t, \mathrm{~d} x)-\lambda_{i}(t) \eta_{i}(\mathrm{~d} x) \mathrm{d} t$ for $\lambda_{i}$ defined in Equation (13) and $\eta_{i}(\mathrm{~d} x)=\mathbb{P}\left(U_{n}^{(i)} \in \mathrm{d} x\right)$.

We characterize the evolution of impulse regime switching securities $S_{i}^{(l)}$ as follows:

$$
\left\{\begin{array}{l}
\mathrm{d} S_{i}^{(l)}(t)=S_{i}^{(l)}(t-)\left[\mu_{i}^{(l)}(t) \mathrm{d} t+\sigma_{i}^{(l)}(t-) \mathrm{d} \bar{\Psi}_{i}^{(l)}(t)\right], \\
S_{i}^{(l)}(0)>0
\end{array}\right.
$$


where the coefficients are determined by the Markov chain J, namely:

$$
\mu_{i}^{(l)}(t):=\left\langle\boldsymbol{\mu}_{i}^{(l)}, J(t)\right\rangle=\sum_{j=1}^{N} \mu_{i, j}^{(l)}\left\langle\mathbf{e}_{j}, J(t)\right\rangle ; \quad \sigma_{i}^{(l)}(t):=\left\langle\sigma_{i}^{(l)}, J(t)\right\rangle=\sum_{j=1}^{N} \sigma_{i, j}^{(l)}\left\langle\mathbf{e}_{j}, J(t)\right\rangle
$$

for $\mu_{i}^{(l)}:=\left(\mu_{i, 1}^{(l)}, \ldots, \mu_{i, N}^{(l)}\right)^{\prime} \in \mathbb{R}^{N}$ and $\sigma_{i}^{(l)}:=\left(\sigma_{i, 1}^{(l)}, \ldots, \sigma_{i, N}^{(l)}\right)^{\prime} \in \mathbb{R}_{+}^{N}(i=1, \ldots, N$ and $l \geq 1)$. The positivity of these asset prices follow from assumption in Equation (11).

Combining Equations (1), (10), (14)-(16), we get an enlarged Itô-Markov additive market:

$$
\left\{\begin{array}{l}
\mathrm{d} B(t)=r(t) B(t) \mathrm{d} t \\
\mathrm{~d} S_{0}(t)=S_{0}(t-)\left[\mu_{0}(t) \mathrm{d} t+\sigma_{0}(t) \mathrm{d} W(t)+\int_{\mathbb{R}} \gamma(t-, x) \bar{\Pi}(\mathrm{d} t, \mathrm{~d} x)+\sum_{i=1}^{N} \int_{\mathbb{R}} x \bar{\Pi}_{U}^{i}(\mathrm{~d} t, \mathrm{~d} x)\right] \\
\mathrm{d} S_{j}(t)=S_{j}(t-)\left[\mu_{j}(t) \mathrm{d} t+\sigma_{j}(t-) \mathrm{d} \bar{\Phi}_{j}(t)\right] \\
\mathrm{d} S^{(k)}(t)=S^{(k)}(t-)\left[\mu^{(k)}(t) \mathrm{d} t+\sigma^{(k)}(t-) \mathrm{d} \bar{X}^{(k)}(t)\right] \\
\mathrm{d} S_{i}^{(l)}(t)=S_{i}^{(l)}(t-)\left[\mu_{i}^{(l)}(t) \mathrm{d} t+\sigma_{i}^{(l)}(t-) \mathrm{d} \bar{\Psi}_{i}^{(l)}(t)\right]
\end{array}\right.
$$

for $i, j=1, \ldots, N, k \geq 2$ and $l \geq 1$. Note that this enlarged market has infinitely many securities. In Section 5 , we prove that under a new martingale measure this market is complete. Note that $\mu_{j}, \mu^{(k)}$, $\mu_{i}^{(l)}, \sigma_{j}, \sigma^{(k)}$ and $\sigma_{i}^{(l)}$ are artificial parameters and can be changed later.

Following Corcuera et al. (2005), the use of power-jump assets of order two can be motivated by a quadratic variation process (Barndorff-Nielsen and Shephard 2003, 2004) and so-called realized variance. Contracts on realized variance are traded regularly on OTC markets. Typically, a 3rd-power-jump asset measures a kind of asymmetry ("skewness") and a 4th-power-jump process measures extremal movements ("kurtosis"). This type of contracts could be also traded. Moreover, one can construct an insurance contracts against a crash based on 4th-power-jump (or ith-power-jump, $i>4$ ) assets. Power-jumps processes appear in other financial-insurance contracts that hedge Lévy jumps as well, e.g., in COS and CDS contracts.

\section{Martingale Measure and Asymptotic Arbitrage}

Considering a financial market containing an infinite number of assets, Kabanov and Kramkov (1994) introduced the notion of large financial market. This type of market is described by a sequence of market models with a finite number of securities each, also called small markets. Kabanov and Kramkov (1994) introduced an extension of the classical approach to arbitrage theory, namely arbitrage in a large financial market, called asymptotic arbitrage. A deep study of asymptotic arbitrage was carried out by Kabanov and Kramkov (1998) and Björk and Näslund (1998).

In this section we identify a martingale measure in our Itô-Markov additive market and prove that this market model is asymptotic-arbitrage-free.

Let us start with the definition of asymptotic arbitrage. Following (Björk and Näslund 1998, Def. 6.1), we say that there is an asymptotic arbitrage opportunity if we have a sequence of strategies such that, for some real number $c>0$, the value process $V^{n}$ on a finite market satisfies:

- $\quad V^{n}(t) \geq-c$ for each $0<t \leq T$ and for each $n \in \mathbb{N}$;

- $V^{n}(0)=0$ for each $n \in \mathbb{N}$;

- $\liminf _{n \rightarrow \infty} V^{n}(T) \geq 0, \mathbb{P}$-a.s; and

- $\mathbb{P}\left(\liminf _{n \rightarrow \infty} V^{n}(T)>0\right)>0$.

Proposition 1 (Björk and Näslund 1998, Prop. 6.1). If there exists a martingale measure $\mathbb{Q}$ equivalent to $\mathbb{P}$, then the market is asymptotic-arbitrage-free. 
In this paper, we formulate the sufficient condition for absence of asymptotic arbitrage in a form of the existence of a martingale measure. Equivalent conditions for the existence of a so-called separated martingale measure can be found in Cuchiero et al. (2016) who introduced No Asymptotic Free Lunch with Vanishing Risk (NALFVR) condition (see also Kreps (1981)).

Now, we find a measure $\mathbb{Q}$ under which the discounted price processes are martingales.

Let $\mathcal{L}^{2}(W)$ be the set of all predictable, $\left\{\mathcal{F}_{t}\right\}$-adapted processes $\xi$ such that $\mathbb{E} \int_{0}^{T} \xi^{2}(s) \mathrm{d} s<\infty$. Similarly, we define $\mathcal{L}^{1}\left(\phi_{j}\right)$, that is, $\xi \in \mathcal{L}^{1}\left(\phi_{j}\right)$ iff $\xi$ is predictable, $\left\{\mathcal{F}_{t}\right\}$-adapted and satisfies $\mathbb{E} \int_{0}^{T}|\xi(s)| \lambda_{j} \mathrm{~d} s<\infty$.

Proposition 2 (Boel and Kohlmann 1980, p. 515). Let $\psi_{0} \in \mathcal{L}^{2}(W), \psi_{j} \in \mathcal{L}^{1}\left(\phi_{j}\right)$ for all $j=1, \ldots, N$ and

$$
\psi_{j}(s)>-1 .
$$

Then,

$$
\begin{aligned}
\ell(t):=\exp \left[\int_{0}^{t} \psi_{0}(s) \mathrm{d} W(s)-\frac{1}{2} \int_{0}^{t} \psi_{0}^{2}(s) \mathrm{d} s-\sum_{j=1}^{N} \int_{0}^{t} \psi_{j}(s) \phi_{j}(\mathrm{~d} s)\right] \\
\times \prod_{j=1}^{N} \prod_{\substack{J(t-) \neq J(t) \\
J(t)=e_{j}}}\left(1+\psi_{j}(t)\right)
\end{aligned}
$$

is a non-negative local martingale. If additionally $\mathbb{E} \ell(t)=1$, then it is a true martingale.

From now on, we assume that

$$
\mathbb{E} \ell(t)=1
$$

Let $\mathbb{Q}$ be the probability measure defined by the Radon-Nikodym derivative

$$
\ell(t)=\left.\frac{\mathrm{d} \mathbb{Q}}{\mathrm{dP}}\right|_{\mathcal{F}_{t}} .
$$

Then, $\ell$, given in Equation (19), is the density process for the new martingale measure $\mathbb{Q}$. By adding a superscript $\mathbb{Q}$, we denote processes observed under this new measure. By a generalized version of Girsanov's theorem for jump-diffusion processes, we have the following theorem:

Theorem 1 (Boel and Kohlmann 1980, p. 517). The process $\bar{X}$ given in Equation (5) under the new martingale measure $\mathbb{Q}$ has the form

$$
\bar{X}^{\mathbb{Q}}(t)=\int_{0}^{t} \sigma_{0}(s) \mathrm{d} W^{\mathbb{Q}}(s)+\int_{0}^{t} \int_{\mathbb{R}} \gamma(s-, x) \bar{\Pi}(\mathrm{d} s, \mathrm{~d} x),
$$

where

$$
W^{\mathbb{Q}}(t)=W(t)-\int_{0}^{t} \psi_{0}(s) \mathrm{d} s
$$

is a standard $\mathbb{Q}$-Brownian motion.

Moreover, for $j=1, \ldots, N$, the process $\bar{\Phi}_{j}$ given in Equation (12) under the measure $\mathbb{Q}$ is a martingale and takes the form

$$
\bar{\Phi}_{j}^{\mathbb{Q}}(t)=\Phi_{j}(t)-\int_{0}^{t}\left(1+\psi_{j}(s)\right) \phi_{j}(\mathrm{~d} s),
$$

that is, the unique predictable projection of $\Phi_{j}$ under $\mathbb{Q}$ is given by

$$
\phi_{j}^{\mathbb{Q}}(t)=\int_{0}^{t}\left(1+\psi_{j}(s)\right) \phi_{j}(\mathrm{~d} s) .
$$


Similarly, for $j=1, \ldots, N$ and $l \geq 1$, the process $\bar{\Psi}_{j}^{(l)}$ given in Equation (12) under the measure $\mathbb{Q}$ is a martingale and takes the form

$$
\bar{\Psi}_{j}^{(l), \mathbb{Q}}(t)=\Psi_{j}^{(l)}(t)-\mathbb{E}\left(U_{n}^{(i)}\right)^{l} \phi_{j}^{\mathbb{Q}}(t) .
$$

Remark 2. If $\psi_{0}$ and $\psi_{j}(j=1, \ldots, N)$ are bounded, then $\mathbb{E} \ell(t)=1$ (see the Novikov condition in Karatzas and Shreve (1998, Cor. 3.5.13, p. 199) and Resnick (2007, Thm. 5.1, p. 135)).

Note that $\bar{X}^{(k)}$ for $k \geq 2$ do not change their laws under the new measure $\mathbb{Q}$. Moreover, under $\mathbb{Q}$, the price processes are represented as follows:

$$
\left\{\begin{array}{l}
\mathrm{d} B(t)=r(t) B(t) \mathrm{d} t \\
\mathrm{~d} S_{0}(t)=S_{0}(t-)\left[\left(\mu_{0}(t)+\sigma_{0}(t) \psi_{0}(t)\right) \mathrm{d} t+\sigma_{0}(t) \mathrm{d} W^{\mathbb{Q}}(t)+\int_{\mathbb{R}} \gamma(t-, x) \bar{\Pi}(\mathrm{d} t, \mathrm{~d} x)+\sum_{i=1}^{N} \iint_{\mathbb{R}} x \bar{\Pi}_{U}^{i}(\mathrm{~d} t, \mathrm{~d} x)\right] \\
\mathrm{d} S_{j}(t)=S_{j}(t-)\left[\left(\mu_{j}(t)+\sigma_{j}(t) \lambda_{j}(t) \psi_{j}(t)\right) \mathrm{d} t+\sigma_{j}(t-) \mathrm{d} \bar{\Phi}_{j}^{\mathbb{Q}}(t)\right] \\
\mathrm{d} S^{(k)}(t)=S^{(k)}(t-)\left[\mu^{(k)}(t) \mathrm{d} t+\sigma^{(k)}(t-) \mathrm{d} \bar{X}^{(k)}(t)\right] \\
\mathrm{d} S_{i}^{(l)}(t)=S_{i}^{(l)}(t-)\left[\mu_{i}^{(l)}(t) \mathrm{d} t+\sigma_{i}^{(l)}(t-) \mathrm{d} \bar{\Psi}_{i}^{(l), \mathbb{Q}}(t)\right]
\end{array}\right.
$$

for $i, j=1, \ldots, N, k \geq 2$ and $l \geq 1$. Note that in the above equation we can take $r(t)=r(t-)$, $\mu_{j}(t)=\mu_{j}(t-)$ and $\sigma_{j}(t)=\sigma_{j}(t-)$ (for $\left.j=0,1, \ldots, N\right)$. In fact, by stochastic integration by parts, the discounted price processes are governed by

$$
\left\{\begin{array}{l}
\mathrm{d} \tilde{S}_{0}(t)=\tilde{S}_{0}(t-)\left[\left(\mu_{0}(t-)+\sigma_{0}(t-) \psi_{0}(t)-r(t-)\right) \mathrm{d} t+\sigma_{0}(t) \mathrm{d} W^{\mathbb{Q}}(t)+\int_{\mathbb{R}} \gamma(t-, x) \bar{\Pi}(\mathrm{d} t, \mathrm{~d} x)\right. \\
\left.\quad+\sum_{i=1}^{N} \int_{\mathbb{R}} x \bar{\Pi}_{U}^{i}(\mathrm{~d} t, \mathrm{~d} x)\right] \\
\mathrm{d} \tilde{S}_{j}(t)=\tilde{S}_{j}(t-)\left[\left(\mu_{j}(t-)+\sigma_{j}(t-) \lambda_{j}(t) \psi_{j}(t)-r(t-)\right) \mathrm{d} t+\sigma_{j}(t-) \mathrm{d} \bar{\Phi}_{j}^{\mathbb{Q}}(t)\right] \\
\mathrm{d} \tilde{S}^{(k)}(t)=\tilde{S}^{(k)}(t-)\left[\left(\mu^{(k)}(t)-r(t)\right) \mathrm{d} t+\sigma^{(k)}(t-) \mathrm{d} \bar{X}^{(k)}(t)\right] \\
\mathrm{d} \tilde{S}_{i}^{(l)}(t)=\tilde{S}_{i}^{(l)}(t-)\left[\left(\mu_{i}^{(l)}(t)-r(t)\right) \mathrm{d} t+\sigma_{i}^{(l)}(t-) \mathrm{d} \bar{\Psi}_{i}^{(l), \mathbb{Q}}(t)\right]
\end{array}\right.
$$

where $\tilde{S}_{0}(t):=B^{-1}(t) S_{0}(t), \tilde{S}_{j}(t):=B^{-1}(t) S_{j}(t), \tilde{S}^{(k)}(t):=B^{-1}(t) S^{(k)}(t)$ and $\tilde{S}_{i}^{(l)}(t):=B^{-1}(t) S_{i}^{(l)}(t)$. Hence, we require $\tilde{S}_{0}, \tilde{S}_{j}, \tilde{S}^{(k)}$ and $\tilde{S}_{i}^{(l)}$ to be local martingales (for $i, j=1, \ldots, N, k \geq 2$ and $l \geq 1$ ). A necessary and sufficient condition for this to hold is given by the following equations:

$$
\left\{\begin{array}{l}
\mu_{0}(t-)+\sigma_{0}(t-) \psi_{0}(t)-r(t-)=0 \\
\mu_{j}(t-)+\sigma_{j}(t-) \lambda_{j}(t) \psi_{j}(t)-r(t-)=0 \\
\mu^{(k)}(t)-r(t)=0 \\
\mu_{i}^{(l)}(t)-r(t)=0 \\
\psi_{j}(t)>-1
\end{array}\right.
$$

for $i, j=1, \ldots, N, k \geq 2$ and $l \geq 1$.

Note that $\lambda_{j}(t)=0$ if $J(t-)=\mathbf{e}_{j}$. Thus, in this case, if $\mu_{j}^{j} \neq r_{j}$, the martingale condition would never be satisfied. Therefore, the discounted price processes of all securities in the enlarged market would not be local martingales under $\mathbb{Q}$. Thus, we have to assume that $\mu_{j}^{j}=r_{j}$ for all $j=1, \ldots, N$ 
to make the market asymptotic-arbitrage-free. From Equation (22), when $\lambda_{j}(t) \neq 0$ (i.e., $\left.J(t-) \neq \mathbf{e}_{j}\right)$, the processes $\psi_{0}$ and $\psi_{j}$ are determined by

$$
\left\{\begin{array}{l}
\psi_{0}(t)=\frac{r(t-)-\mu_{0}(t-)}{\sigma_{0}(t-)}, \\
\psi_{j}(t)=\frac{r(t-)-\mu_{j}(t-)}{\sigma_{j}(t-) \lambda_{j}(t)}, \quad j=1, \ldots, N,
\end{array}\right.
$$

where we assume that

$$
r(t-)>\mu_{j}(t-)-\sigma_{j}(t-) \lambda_{j}(t)
$$

to satisfy the condition in Equation (18).

Note that $\psi_{0}$ and $\psi_{j}(j=1, \ldots, N)$ are bounded. Hence, by Remark 2, the density process $\ell$ is a true martingale. Note that $\psi_{j}(j=1, \ldots, N)$ satisfies the assumptions of Proposition 2. We can only determine $\psi_{j}$ when $J(t-) \neq \mathbf{e}_{j}$ for $j=1, \ldots, N$ but this is sufficient to determine the equivalent martingale measure $\mathbb{Q}$. Indeed, if $J(t-)=\mathbf{e}_{j}$ for $j=1, \ldots, N$, then $\phi_{j}(t)=0$ and $\psi_{j}$ has no influence on the value of the right side of Equation (19). The above analysis yields the following theorem.

Theorem 2. Assume that $\mu_{j}^{j}=r_{j}$ for all $j=1, \ldots, N$ and $\psi_{0}$ and $\psi_{j}$ are given by Equation (23). Then, the discounted price processes of the securities in the enlarged market (Equation (21)) are local martingales under $\mathbb{Q}$ and this market is asymptotic-arbitrage-free.

From now on, we assume that $\mu_{j}^{j}=r_{j}$ for all $j=1, \ldots, N$.

\section{Asymptotic Completeness of the Enlarged Market}

Now, we analyze asymptotic completeness of the enlarged Itô-Markov additive market. A market is said to be complete if each claim can be replicated by a strategy, that is, the claim can be represented as a stochastic integral with respect to the asset prices. We take as class of contingent claims the set $L^{2}(\Omega, \mathcal{F}, \mathbb{Q})$ of square integrable random variables under the equivalent martingale measure; then, a self-financing strategy is represented as an integrable process and the value of a self-financing portfolio is represented as the stochastic integral of the strategy with respect to the assets. In the case of market models with an infinite number of assets, we define completeness in terms of approximate replication of claims.

For finite market asset, completeness is equivalent to uniqueness of the equivalent martingale measure. In the case of large markets, this property does not occur. Artzner and Heath (1995) constructed a financial market with countably many securities for which there are two equivalent martingale measures under which the market is approximately complete. In the context of a large financial market, Bättig (1999) and Bättig and Jarrow (1999) suggested a definition of completeness that uses neither the notion of arbitrage-free nor equivalent martingale measures. Bättig (1999) constructed an example showing that the existence of an equivalent martingale measure excludes the possibility of replicating a claim, hence proving that the notions of arbitrage-free and completeness could be unrelated to each other in daily practice. 
Under $\mathbb{Q}$, the price processes of the securities in the arbitrage-free market have the following representations:

$$
\left\{\begin{array}{l}
\mathrm{d} B(t)=r(t) B(t) \mathrm{d} t, \\
\mathrm{~d} S_{0}(t)=S_{0}(t-)\left[r(t) \mathrm{d} t+\sigma_{0}(t) \mathrm{d} W^{\mathbb{Q}}(t)+\int_{\mathbb{R}} \gamma(t-, x) \bar{\Pi}(\mathrm{d} t, \mathrm{~d} x)+\sum_{i=1}^{N} \int_{\mathbb{R}} x \bar{\Pi}_{U}^{i}(\mathrm{~d} t, \mathrm{~d} x)\right], \\
\mathrm{d} S_{j}(t)=S_{j}(t-)\left[r(t) \mathrm{d} t+\sigma_{j}(t-) \mathrm{d} \bar{\Phi}_{j}^{\mathbb{Q}}(t)\right], \\
\mathrm{d} S^{(k)}(t)=S^{(k)}(t-)\left[r(t) \mathrm{d} t+\sigma^{(k)}(t-) \mathrm{d} \bar{X}^{(k)}(t)\right], \\
\mathrm{d} S_{i}^{(l)}(t)=S_{i}^{(l)}(t-)\left[r(t) \mathrm{d} t+\sigma_{i}^{(l)}(t-) \mathrm{d} \bar{\Psi}_{i}^{(l), \mathbb{Q}}(t)\right],
\end{array}\right.
$$

for $i, j=1, \ldots, N, k \geq 2$ and $l \geq 1$.

We show that the enlarged market (Equation (25)) is asymptotically complete in the sense that for every square-integrable contingent claim $A$ (i.e., a non-negative square-integrable random variable in $L^{2}(\Omega, \mathcal{F}, \mathbb{Q})$ ) we can set up a sequence of self-financing portfolios whose final values converge in $L^{2}(\Omega, \mathcal{F}, \mathbb{Q})$ to $A$.

These portfolios consist of a finite number of risk-free asset, risky asset, $k$ th-power-jump assets, jth geometric Markovian jump security and impulse regime switching securities. We use the following martingale representation property.

Theorem 3 (Palmowski et al. 2018). Any square-integrable, $\left\{\mathcal{F}_{t}\right\}$-adapted $\mathbb{Q}$-martingale $M$ can be represented as follows:

$$
\begin{aligned}
M(t)= & M(0)+\int_{0}^{t} h_{0}(s) \mathrm{d} X^{\mathbb{Q}}(s)+\sum_{j=1}^{N} \int_{0}^{t} h_{j}(s) \mathrm{d} \bar{\Phi}_{j}^{\mathbb{Q}}(s)+\sum_{k=2}^{\infty} \int_{0}^{t} h^{(k)}(s) \mathrm{d} \bar{X}^{(k)}(s) \\
& +\sum_{i=1}^{N} \sum_{l=1}^{\infty} \int_{0}^{t} h_{i}^{(l)}(s) \mathrm{d} \bar{\Psi}_{i}^{(l), \mathbb{Q}}(s)
\end{aligned}
$$

where $h_{0}, h_{j}, h^{(k)}$ and $h_{i}^{(l)}$ are predictable processes (for $i, j=1, \ldots, N, k \geq 2$ and $l \geq 1$ ).

Remark 3. The right-hand side of Equation (26) is understood as follows. We take finite sums

$$
\sum_{k=2}^{K} \int_{0}^{t} h^{(k)}(s) \mathrm{d} \bar{X}^{(k)}(s) \text { and } \sum_{l=1}^{K} \int_{0}^{t} h_{i}^{(l)}(s) \mathrm{d} \bar{\Psi}_{i}^{(l), \mathbb{Q}}(s)
$$

in $L^{2}(\Omega, \mathcal{F}, \mathbb{Q})$. Since $L^{2}(\Omega, \mathcal{F}, \mathbb{Q})$ is a Hilbert space, the right-hand side of Equation (26) is understood as the limit of the above expressions in $L^{2}(\Omega, \mathcal{F}, \mathbb{Q})$ as $K \rightarrow \infty$.

We are ready to prove the main result of this section.

Theorem 4. The market (Equation (25)) under $\mathbb{Q}$ is asymptotically complete.

Proof. We consider a square-integrable contingent claim $A$ with maturity $T$. Let

$$
M(t):=E_{\mathbb{Q}}\left[\exp \left(-\int_{0}^{T} r(s) \mathrm{d} s\right) A \mid \mathcal{F}_{t}\right]
$$


and

$$
\begin{aligned}
M^{K}(t):= & M^{K}(0)+\int_{0}^{t} h_{0}(s) \mathrm{d} X^{\mathbb{Q}}(s)+\sum_{j=1}^{N} \int_{0}^{t} h_{j}(s) \mathrm{d} \bar{\Phi}_{j}^{\mathbb{Q}}(s)+\sum_{k=2}^{K} \int_{0}^{t} h^{(k)}(s) \mathrm{d} \bar{X}^{(k)}(s) \\
& +\sum_{i=1}^{N} \sum_{l=1}^{K} \int_{0}^{t} h_{i}^{(l)}(s) \mathrm{d} \bar{\Psi}_{i}^{(l), \mathbb{Q}}(s) .
\end{aligned}
$$

By the martingale representation property given in Theorem 3, we see that

$$
\lim _{K \rightarrow \infty} M^{K}(t)=M(t)
$$

in $L^{2}(\Omega, \mathcal{F}, \mathbb{Q})$. For $K \geq 2$, we introduce the sequence of portfolios

$$
\theta^{K}(t):=\left(\alpha^{K}(t), \beta_{0}(t), \beta_{1}(t), \ldots, \beta_{N}(t), \beta^{(2)}(t), \ldots, \beta^{(K)}(t), \beta_{1}^{(1)}(t), \ldots, \beta_{N}^{(K)}(t)\right) .
$$

We assume that all processes in $\theta^{K}$ are predictable and

$$
\begin{gathered}
E_{\mathbb{Q}} \int_{0}^{t}\left(\alpha^{K}(s)\right)^{2} \mathrm{~d} s<\infty, \quad E_{\mathbb{Q}} \int_{0}^{t}\left(\beta_{0}(s)\right)^{2} \mathrm{~d}\left\langle S_{0}\right\rangle(s)<\infty, \quad E_{\mathbb{Q}} \int_{0}^{t}\left(\beta_{j}(s)\right)^{2} \mathrm{~d}\left\langle S_{j}\right\rangle(s)<\infty, \\
E_{\mathbb{Q}} \int_{0}^{t}\left(\beta^{(k)}(s)\right)^{2} \mathrm{~d}\left\langle S^{(k)}\right\rangle(s)<\infty, \quad E_{\mathbb{Q}} \int_{0}^{t}\left(\beta_{i}^{(l)}(s)\right)^{2} \mathrm{~d}\left\langle S_{i}^{(l)}\right\rangle(s)<\infty .
\end{gathered}
$$

Here, $\alpha^{K}$ corresponds to the number of risk-free assets, $\beta_{0}$ is the number of stocks, $\beta_{j}(j=1, \ldots, N)$ is the number of units of the $j$ th geometric Markovian jump security, $\beta^{(k)}(k=2, \ldots, K)$ is the number of assets $S^{(k)}$, and $\beta_{i}^{(l)}(i=1, \ldots, N, l=1, \ldots, K)$ is the number of assets $S_{i}^{(l)}$.

We construct the portfolio $\theta^{K}$ as follows:

$$
\begin{aligned}
\alpha^{K}(t) & :=M^{K}(t-)-\beta_{0}(t) B^{-1}(t) S_{0}(t-)-\sum_{j=1}^{N} \beta_{j}(t) B^{-1}(t) S_{j}(t-) \\
& -\sum_{k=2}^{K} \beta^{(k)}(t) B^{-1}(t) S^{(k)}(t-)-\sum_{i=1}^{N} \sum_{l=1}^{K} \beta_{i}^{(l)}(t) B^{-1}(t) S_{i}^{(l)}(t-) \\
\beta_{0}(t) & :=h_{0}(t) B(t) S_{0}^{-1}(t-), \\
\beta_{j}(t) & :=\frac{h_{j}(t)}{\sigma_{j}(t-)} B(t) S_{j}^{-1}(t-), \\
\beta^{(k)}(t) & :=\frac{h^{(k)}(t)}{\sigma^{(k)}(t-)} B(t)\left(S^{(k)}\right)^{-1}(t-) \\
\beta_{i}^{(l)}(t) & :=\frac{h_{i}^{(l)}(t)}{\sigma_{i}^{(l)}(t-)} B(t)\left(S_{i}^{(l)}\right)^{-1}(t-) .
\end{aligned}
$$

Observe that for this choice of portfolio, the moment conditions in Equations (29) and (30) are satisfied, hence all stochastic integrals for this portfolio are well-defined. For example, we prove that

$$
E_{\mathbb{Q}} \int_{0}^{t}\left(\beta_{0}(s)\right)^{2} \mathrm{~d}\left\langle S_{0}\right\rangle(s)<\infty .
$$

The proofs of other conditions are similar. To show (32) note that

$$
d\left\langle S_{0}\right\rangle(s)=S_{0}^{2}(t-)\left[\sigma_{0}^{2}(t) \mathrm{d} t+\int_{\mathbb{R}} \gamma^{2}(t-, x) \bar{\Pi}(\mathrm{d} t, \mathrm{~d} x)+\sum_{i=1}^{N} \int_{\mathbb{R}} x^{2} \bar{\Pi}_{U}^{i}(\mathrm{~d} t, \mathrm{~d} x)\right]
$$


and this condition is equivalent to requirement that

$$
\begin{gathered}
\int_{0}^{\infty} h_{0}^{2}(t) e^{2 r(t)} \sigma_{0}^{2}(t) \mathrm{d} t<\infty, \quad \int_{0}^{\infty} h_{0}^{2}(t) e^{2 r(t)} \lambda_{i}(t) \int_{\mathbb{R}} \gamma^{2}(t-, x) v(\mathrm{~d} x) \mathrm{d} t<\infty, \\
\int_{\mathbb{R}} x^{2} \eta_{i}(\mathrm{~d} x) \int_{0}^{\infty} h_{0}^{2}(t) e^{2 r(t)} \lambda_{i}(t) \mathrm{d} t<\infty .
\end{gathered}
$$

The above conditions follow from Theorem 3 because $\int_{0}^{\infty} h_{0}^{2}(t) \mathrm{d} t<\infty$. Note that,

$$
\begin{aligned}
\Delta M^{K}(t) & =h_{0}(t) \Delta X^{\mathbb{Q}}(t)+\sum_{j=1}^{N} h_{j}(t) \Delta \bar{\Phi}_{j}^{\mathbb{Q}}(t)+\sum_{k=2}^{K} h^{(k)}(t) \Delta \bar{X}^{(k)}(t)+\sum_{i=1}^{N} \sum_{l=1}^{K} h_{i}^{(l)}(t) \Delta \bar{\Psi}_{i}^{(l), \mathbb{Q}}(t), \\
\Delta S_{0}(t) & =S_{0}(t-) \Delta X^{\mathbb{Q}}(t), \\
\Delta S_{j}(t) & =S_{j}(t-) \sigma_{j}(t-) \Delta \bar{\Phi}_{j}^{\mathbb{Q}}(t), \\
\Delta S^{(k)}(t) & =S^{(k)}(t-) \sigma^{(k)}(t-) \Delta \bar{X}^{(k)}(t), \quad \Delta S_{i}^{(l)}(t)=S_{i}^{(l)}(t-) \sigma_{i}^{(l)}(t-) \Delta \bar{\Psi}_{i}^{(l), \mathbb{Q}}(t) .
\end{aligned}
$$

We claim that $\left\{\theta^{K}, K \geq 2\right\}$ is the sequence of self-financing portfolios which replicates $A$. Indeed, by Equations (31) and (33), the value $V^{K}$ of the portfolio $\theta^{K}$ is expressed by

$$
\begin{aligned}
V^{K}(t)= & \alpha^{K}(t) B(t)+\beta_{0}(t) S_{0}(t)+\sum_{j=1}^{N} \beta_{j}(t) S_{j}(t)+\sum_{k=2}^{K} \beta^{(k)}(t) S^{(k)}(t)+\sum_{i=1}^{N} \sum_{l=1}^{K} \beta_{i}^{(l)}(t) S_{i}^{(l)}(t) \\
= & M^{K}(t) B(t)-\Delta M^{K}(t) B(t)+\beta_{0}(t) \Delta S_{0}(t)+\sum_{j=1}^{N} \beta_{j}(t) \Delta S_{j}(t)+\sum_{k=2}^{K} \beta^{(k)}(t) \Delta S^{(k)}(t) \\
& +\sum_{i=1}^{N} \sum_{l=1}^{K} \beta_{i}^{(l)}(t) \Delta S_{i}^{(l)}(t)=M^{K}(t) B(t) .
\end{aligned}
$$

Thus, the sequence of portfolios $\left\{\theta^{K}, K \geq 2\right\}$ replicates the claim $A$. We denote

$$
\begin{aligned}
G^{K}(u):= & \int_{0}^{u} \alpha^{K}(t) \mathrm{d} B(t)+\int_{0}^{u} \beta_{0}(t) \mathrm{d} S_{0}(t)+\sum_{j=1}^{N} \int_{0}^{u} \beta_{j}(t) \mathrm{d} S_{j}(t) \\
& +\sum_{k=2}^{K} \int_{0}^{u} \beta^{(k)}(t) \mathrm{d} S^{(k)}(t)+\sum_{i=1}^{N} \sum_{l=1}^{K} \int_{0}^{u} \beta_{i}^{(l)}(t) \mathrm{d} S_{i}^{(l)}(t)
\end{aligned}
$$

the gain process, i.e., the gains or losses obtained up to time $u$ by following $\theta^{K}$. We show

$$
G^{K}(u)+M^{K}(0)=M^{K}(u) B(u),
$$

which implies that the portfolio is self-financing. Note that, from Equation (28), we have

$$
\lim _{K \rightarrow \infty} G^{K}(u)=\lim _{K \rightarrow \infty} M^{K}(u) B(u)-\lim _{K \rightarrow \infty} M^{K}(0)=M(u) B(u)-M(0) .
$$


Thus, the portfolios with infinitely many assets are self-financing as well. Inserting Equation (31) into Equation (34), we derive

$$
\begin{aligned}
& G^{K}(u)=\int_{0}^{u} M^{K}(t-) \mathrm{d} B(t)-\int_{0}^{u} h_{0}(t) \mathrm{d} B(t)-\sum_{k=2}^{K} \int_{0}^{u} \frac{h^{(k)}(t)}{\sigma^{(k)}(t-)} \mathrm{d} B(t) \\
& -\sum_{j=1}^{N} \int_{0}^{u} \frac{h_{j}(t)}{\sigma_{j}(t-)} \mathrm{d} B(t)-\sum_{i=1}^{N} \sum_{l=1}^{K} \int_{0}^{u} \frac{h_{i}^{(l)}(t)}{\sigma_{i}^{(l)}(t-)} \mathrm{d} B(t)+\int_{0}^{u} h_{0}(t) B(t) S_{0}^{-1}(t-) \mathrm{d} S_{0}(t) \\
& +\sum_{j=1}^{N} \int_{0}^{u} \frac{h_{j}(t)}{\sigma_{j}(t-)} B(t) S_{j}^{-1}(t-) \mathrm{d} S_{j}(t)+\sum_{k=2}^{K} \int_{0}^{u} \frac{h^{(k)}(t)}{\sigma^{(k)}(t-)} B(t)\left(S^{(k)}\right)^{-1}(t-) \mathrm{d} S^{(k)}(t) \\
& +\sum_{i=1}^{N} \sum_{l=1}^{K} \int_{0}^{u} \frac{h_{i}^{(l)}(t)}{\sigma_{i}^{(l)}(t-)} B(t)\left(S_{i}^{(l)}\right)^{-1}(t-) \mathrm{d} S_{i}^{(l)}(t) .
\end{aligned}
$$

From the martingale representation property given in Theorem 3, the first component of the above sum has the form

$$
\begin{aligned}
& \int_{0}^{u} M^{K}(t-) \mathrm{d} B(t)=\int_{0}^{u}\left(M^{K}(0)+\int_{0}^{t-} h_{0}(s) \mathrm{d} X^{\mathbb{Q}}(s)+\sum_{j=1}^{N} \int_{0}^{t-} h_{j}(s) \mathrm{d} \bar{\Phi}_{j}^{\mathbb{Q}}(s)\right. \\
& \left.\quad+\sum_{k=2}^{K} \int_{0}^{t-} h^{(k)}(s) \mathrm{d} \bar{X}^{(k)}(s)+\sum_{i=1}^{N} \sum_{l=1}^{K} \int_{0}^{t-} h_{i}^{(l)}(s) \mathrm{d} \bar{\Psi}_{i}^{(l) \mathbb{Q}}(s)\right) \mathrm{d} B(t) \\
& =M^{K}(0)(B(u)-B(0))+\int_{0}^{u} h_{0}(s)(B(u)-B(s)) \mathrm{d} X^{\mathbb{Q}}(s)+\sum_{j=1}^{N} \int_{0}^{u} h_{j}(s)(B(u)-B(s)) \mathrm{d} \bar{\Phi}_{j}^{\mathbb{Q}}(s) \\
& \quad+\sum_{k=2}^{K} \int_{0}^{u} h^{(k)}(s)(B(u)-B(s)) \mathrm{d} \bar{X}^{(k)}(s)+\sum_{i=1}^{N} \sum_{l=1}^{K} \int_{0}^{u} h_{i}^{(l)}(s)(B(u)-B(s)) \mathrm{d} \bar{\Psi}_{i}^{(l), \mathbb{Q}(s) .}
\end{aligned}
$$

Now, using Equation (27) and fact that $B(0)=1$, we can rewrite the above as follows:

$$
\begin{gathered}
\int_{0}^{u} M^{K}(t-) \mathrm{d} B(t)=M^{K}(0)(B(u)-B(0))+B(u)\left(M^{K}(u)-M^{K}(0)\right)-\int_{0}^{u} h_{0}(s) B(s) \mathrm{d} \bar{X}^{\mathbb{Q}}(s) \\
\quad-\sum_{j=1}^{N} \int_{0}^{u} h_{j}(s) B(s) \mathrm{d} \bar{\Phi}_{j}^{\mathbb{Q}}(s)-\sum_{k=2}^{K} \int_{0}^{u} h^{(k)}(s) B(s) \mathrm{d} \bar{X}^{(k)}(s)-\sum_{i=1}^{N} \sum_{l=1}^{K} \int_{0}^{u} h_{i}^{(l)}(s) B(s) \mathrm{d} \bar{\Psi}_{i}^{(l), \mathbb{Q}}(s) \\
=M^{K}(u) B(u)-M^{K}(0)-\int_{0}^{u} h_{0}(s) B(s) \mathrm{d} X^{\mathbb{Q}}(s)-\sum_{j=1}^{N} \int_{0}^{u} h_{j}(s) B(s) \mathrm{d} \bar{\Phi}_{j}^{\mathbb{Q}}(s) \\
\quad-\sum_{k=2}^{K} \int_{0}^{u} h^{(k)}(s) B(s) \mathrm{d} \bar{X}^{(k)}(s)-\sum_{i=1}^{N} \sum_{l=1}^{K} \int_{0}^{u} h_{i}^{(l)}(s) B(s) \mathrm{d} \bar{\Psi}_{i}^{(l), \mathbb{Q}}(s) .
\end{gathered}
$$


Inserting the above equality into Equation (36), the gain process can be written as:

$$
\begin{aligned}
G^{K}(u) & =M^{K}(u) B(u)-M^{K}(0)-\int_{0}^{u} h_{0}(t) B(t) \mathrm{d} X^{\mathbb{Q}}(t)-\sum_{j=1}^{N} \int_{0}^{u} h_{j}(t) B(t) \mathrm{d} \bar{\Phi}_{j}^{\mathbb{Q}}(t) \\
& -\sum_{k=2}^{K} \int_{0}^{u} h^{(k)}(t) B(t) \mathrm{d} \bar{X}^{(k)}(t)-\sum_{i=1}^{N} \sum_{l=1}^{K} \int_{0}^{u} h_{i}^{(l)}(t) B(t) \mathrm{d} \bar{\Psi}_{i}^{(l), \mathbb{Q}}(t)-\int_{0}^{u} h_{0}(t) \mathrm{d} B(t) \\
& -\sum_{k=2}^{K} \int_{0}^{u} \frac{h^{(k)}(t)}{\sigma^{(k)}(t-)} \mathrm{d} B(t)-\sum_{j=1}^{N} \int_{0}^{u} \frac{h_{j}(t)}{\sigma_{j}(t-)} \mathrm{d} B(t)-\sum_{i=1}^{N} \sum_{l=1}^{K} \int_{0}^{u} \frac{h_{i}^{(l)}(t)}{\sigma_{i}^{(l)}(t-)} \mathrm{d} B(t) \\
& +\int_{0}^{u} h_{0}(t) B(t) S_{0}^{-1}(t-) \mathrm{d} S_{0}(t)+\sum_{j=1}^{N} \int_{0}^{u} \frac{h_{j}(t)}{\sigma_{j}(t-)} B(t) S_{j}^{-1}(t-) \mathrm{d} S_{j}(t) \\
& +\sum_{k=2}^{K} \int_{0}^{u} \frac{h^{(k)}(t)}{\sigma^{(k)}(t-)} B(t)\left(S^{(k)}\right)^{-1}(t-) \mathrm{d} S^{(k)}(t)+\sum_{i=1}^{N} \sum_{l=1}^{K} \int_{0}^{u} \frac{h_{i}^{(l)}(t)}{\sigma_{i}^{(l)}(t-)} B(t)\left(S_{i}^{(l)}\right)^{-1}(t-) \mathrm{d} S_{i}^{(l)}(t) \\
= & M^{K}(u) B(u)-M(0) .
\end{aligned}
$$

Thus, Equation (35) holds true and the portfolio $\theta^{K}$ is self-financing.

\section{Optimal Portfolio Selection in an Itô-Markov Additive Market}

In this section we solve the optimization problem related to identifying the optimal strategy that maximizes the expected value of the utility function of the wealth process at the end of some fixed period. The analysis is conducted for the logarithmic and power utility functions.

Recall that our Itô-Markov additive market is given by Equation (17). Equations (15) and (16) can be rewritten as follows:

$$
\begin{aligned}
\mathrm{d} S^{(k)}(t) & =S^{(k)}(t-)\left[\mu^{(k)}(t) \mathrm{d} t+\int_{\mathbb{R}} \sigma^{(k)}(t-) \gamma^{k}(t-, x) \bar{\Pi}(\mathrm{d} t, \mathrm{~d} x)\right], \\
\mathrm{d} S_{i}^{(l)}(t) & =S_{i}^{(l)}(t-)\left[\mu_{i}^{(l)}(t) \mathrm{d} t+\int_{\mathbb{R}} x^{l} \sigma_{i}^{(l)}(t-) \bar{\Pi}_{U}^{i}(\mathrm{~d} t, \mathrm{~d} x)\right]
\end{aligned}
$$

for $i=1, \ldots, N, k \geq 2$ and $l \geq 1$. Note that we consider the price processes with respect the original probability measure $\mathbb{P}$.

We restrict ourselves to self-financing portfolio strategies. Denote by $\pi_{0}$ the proportion of wealth invested in stock. Let $\pi_{j}(j=1, \ldots, N), \pi^{(k)}(k \geq 2)$ and $\pi_{i}^{(l)}(i=1, \ldots, N, l \geq 1)$ be the proportions of wealth invested in the $j$ th geometric Markovian jump security $S_{j}$, in the Markovian power-jump securities $S^{(k)}$ and in the impulse regime switching securities $S_{i}^{(l)}$, respectively. The balance of the investor's wealth is invested in the risk-free asset. We denote by $\pi(t)=$ $\left(\pi_{0}(t), \pi_{1}(t), \ldots, \pi_{N}(t), \pi^{(2)}(t), \ldots, \pi_{1}^{(1)}(t), \pi_{2}^{(1)}(t), \ldots\right)$ a portfolio strategy. We do allow short selling, but we assume that the wealth process is nonnegative at any instant (Teplá 2000). 
Let $K<\infty$ be the number of different assets held by the investor in his portfolio. The wealth process $R_{\pi}^{K}$ for the first $K$ assets is governed by the following stochastic differential equation (for $t \in[0, T]$ ):

$$
\begin{aligned}
\frac{\mathrm{d} R_{\pi}^{K}(t)}{R_{\pi}^{K}(t-)}:= & \left(r(t)+\sum_{j=0}^{N} \pi_{j}(t)\left(\mu_{j}(t)-r(t)\right)+\sum_{k=2}^{K} \pi^{(k)}(t)\left(\mu^{(k)}(t)-r(t)\right)\right. \\
& \left.+\sum_{i=1}^{N} \sum_{l=1}^{K} \pi_{i}^{(l)}(t)\left(\mu_{i}^{(l)}(t)-r(t)\right)\right) \mathrm{d} t+\pi_{0}(t) \sigma_{0}(t-) \mathrm{d} W(t)+\sum_{j=1}^{N} \pi_{j}(t) \sigma_{j}(t-) \mathrm{d} \bar{\Phi}_{j}(t) \\
& +\int_{\mathbb{R}}\left(\pi_{0}(t) \gamma(t-, x)+\sum_{k=2}^{K} \pi^{(k)}(t) \sigma^{(k)}(t-) \gamma^{k}(t-, x)\right) \bar{\Pi}(\mathrm{d} t, \mathrm{~d} x) \\
& +\sum_{i=1}^{N} \int_{\mathbb{R}}\left(x \pi_{0}(t)+\sum_{l=1}^{K} x^{l} \pi_{i}^{(l)}(t) \sigma_{i}^{(l)}(t-)\right) \bar{\Pi}_{U}^{i}(\mathrm{~d} t, \mathrm{~d} x) .
\end{aligned}
$$

Note that in Equation (37) we can take $r(t)=r(t-), \mu_{j}(t)=\mu_{j}(t-)(j=0,1, \ldots, N), \mu^{(k)}(t)=$ $\mu^{(k)}(t-)(k \geq 2)$ and $\mu_{i}^{(l)}(t)=\mu_{i}^{(l)}(t-)(i=1, \ldots, N, l \geq 1)$.

Let $\mathcal{A}$ be the class of admissible portfolio strategies $\pi$ such that $\pi$ is predictable, $R_{\pi}^{K}>0, \int_{0}^{T}|\pi(t)|^{2} \mathrm{~d} t<\infty \mathbb{P}$ - a.s., $\int_{\mathbb{R}}\left(\pi_{0}(t) \gamma(t-, x)+\sum_{k=2}^{K} \pi^{(k)}(t) \sigma^{(k)}(t-) \gamma^{k}(t-, x)\right) \bar{\Pi}(\mathrm{d} t, \mathrm{~d} x)<\infty$, $\int_{\mathbb{R}} \sum_{i=1}^{N}\left(x \pi_{0}(t)+\sum_{l=1}^{K} x^{l} \pi_{i}^{(l)}(t) \sigma_{i}^{(l)}(t-)\right) \bar{\Pi}_{U}^{i}(\mathrm{~d} t, \mathrm{~d} x)<\infty$ and $\pi$ satisfies the following convergence: the wealth process $R_{\pi}^{K}$ converges to a process $R_{\pi}$ in $L^{2}(\Omega, \mathcal{F}, \mathbb{P})$, where $R_{\pi}^{K}$ is the solution of the SDE in Equation (37) (see Itô's formula in Protter (2005, Thm. 32)), that is,

$$
\begin{aligned}
R_{\pi}^{K}(t)= & R_{\pi}^{K}(0) \exp \left[\int _ { 0 } ^ { t } \left(r(s-)+\sum_{j=0}^{N} \pi_{j}(s)\left(\mu_{j}(s-)-r(s-)\right)+\sum_{k=2}^{K} \pi^{(k)}(s)\left(\mu^{(k)}(s)-r(s-)\right)\right.\right. \\
& \left.+\sum_{i=1}^{N} \sum_{l=1}^{K} \pi_{i}^{(l)}(s)\left(\mu_{i}^{(l)}(s)-r(s-)\right)-\frac{1}{2} \pi_{0}^{2}(s) \sigma_{0}^{2}(s-)\right) \mathrm{d} s \\
& +\int_{0}^{t} \pi_{0}(s) \sigma_{0}(s-) d W(s)+\sum_{j=1}^{N} \int_{0}^{t}\left(\log \left(1+\pi_{j}(s) \sigma_{j}(s-)\right)-\pi_{j}(s) \sigma_{j}(s-)\right) \lambda_{j}(s) d s \\
& +\int_{0}^{t} \int_{\mathbb{R}} \log \left(1+\pi_{0}(s) \gamma(s-, x)+\sum_{k=2}^{K} \pi^{(k)}(s) \sigma^{(k)}(s-) \gamma^{k}(s-, x)\right) \bar{\Pi}(\mathrm{d} s, \mathrm{~d} x) \\
& +\int_{0}^{t} \int_{\mathbb{R}}\left(\log \left(1+\pi_{0}(s) \gamma(s-, x)+\sum_{k=2}^{K} \pi^{(k)}(s) \sigma^{(k)}(s-) \gamma^{k}(s-, x)\right)-\pi_{0}(s) \gamma(s-, x)\right. \\
& \left.-\sum_{k=2}^{K} \pi^{(k)}(s) \sigma^{(k)}(s-) \gamma^{k}(s-, x)\right) v(\mathrm{~d} x) \mathrm{d} s+\sum_{j=1}^{N} \int_{0}^{t} \log \left(1+\pi_{j}(s) \sigma_{j}(s-)\right) \mathrm{d} \bar{\Phi}_{j}(s) \\
& +\sum_{i=1}^{N} \int_{0}^{t} \int_{\mathbb{R}} \log \left(1+x \pi_{0}(s)+\sum_{l=1}^{K} \pi_{i}^{(l)}(s) \sigma_{i}^{(l)}(s-) x^{l}\right) \bar{\Pi}_{U}^{i}(\mathrm{~d} s, \mathrm{~d} x) \\
& +\sum_{i=1}^{N} \int_{0}^{t} \int_{\mathbb{R}}\left(\log \left(1+x \pi_{0}(s)+\sum_{l=1}^{K} \pi_{i}^{(l)}(s) \sigma_{i}^{(l)}(s-) x^{l}\right)-x \pi_{0}(s)\right. \\
& \left.\left.-\sum_{l=1}^{K} \pi_{i}^{(l)}(s) \sigma_{i}^{(l)}(s-) x^{l}\right) \lambda_{i}(s) \eta(\mathrm{d} x) \mathrm{d} s\right] .
\end{aligned}
$$

In other words, for $\pi \in \mathcal{A}$, we require that

$$
\lim _{K \rightarrow \infty} R_{\pi}^{K}(t)=R_{\pi}(t)
$$


in $L^{2}(\Omega, \mathcal{F}, \mathbb{P})$.

Remark 4. Note that Equation (38) holds true if

$$
\mathbb{E}_{t, z, i}\left(R_{\pi}^{K}(t)\right)^{2}<\infty, \mathbb{E}_{t, z, i}\left(R_{\pi}(t)\right)^{2}<\infty,
$$

and

$$
\begin{gathered}
\mathbb{E}_{t, z, i}\left|\int_{0}^{t} \sum_{k=K+1}^{\infty} \pi^{(k)}(s)\left(\mu^{(k)}(s)-r(s-)\right) \mathrm{d} s\right|^{2}, \\
\mathbb{E}_{t, z, i}\left|\int_{0}^{t} \sum_{i=1}^{N} \sum_{l=K+1}^{\infty} \pi_{i}^{(l)}(s)\left(\mu_{i}^{(l)}(s)-r(s-)\right) \mathrm{d} s\right|^{2}, \\
\mathbb{E}_{t, z, i} \mid \int_{0}^{t} \int_{\mathbb{R}} \log \left(\left.\frac{1+\pi_{0}(s) \gamma(s-, x)+\sum_{k=2}^{\infty} \pi^{(k)}(s) \sigma^{(k)}(s-) \gamma^{k}(s-, x)}{1+\pi_{0}(s) \gamma(s-, x)+\sum_{k=2}^{K} \pi^{(k)}(s) \sigma^{(k)}(s-) \gamma^{k}(s-, x)} \bar{\Pi}(\mathrm{d} s, \mathrm{~d} x)\right|^{2},\right. \\
\mathbb{E}_{t, z, i} \mid \int_{0}^{t} \int_{\mathbb{R}}\left(\log \left(\frac{1+\pi_{0}(s) \gamma(s-, x)+\sum_{k=2}^{\infty} \pi^{(k)}(s) \sigma^{(k)}(s-) \gamma^{k}(s-, x)}{1+\pi_{0}(s) \gamma(s-, x)+\sum_{k=2}^{K} \pi^{(k)}(s) \sigma^{(k)}(s-) \gamma^{k}(s-, x)}\right)\right. \\
\left.-\sum_{k=K+1}^{\infty} \pi^{(k)}(s) \sigma^{(k)}(s-) \gamma^{k}(s-, x)\right)\left.v(\mathrm{~d} x) \mathrm{d} s\right|^{2} \\
\mathbb{E}_{t, z, i}\left|\int_{0}^{t} \int_{\mathbb{R}} \log \left(\frac{1+x \pi_{0}(s)+\sum_{l=2}^{\infty} \pi_{i}^{(l)}(s) \sigma_{i}^{(l)}(s-) x^{l}}{1+x \pi_{0}(s)+\sum_{l=2}^{K} \pi_{i}^{(l)}(s) \sigma_{i}^{(l)}(s-) x^{l}}\right)^{i}(\mathrm{~d} s, \mathrm{~d} x)\right|^{2}, \\
\mathbb{E}_{t, z, i} \mid \sum_{i=1}^{N} \int_{0}^{t} \int_{\mathbb{R}}\left(\log \left(\frac{1+x \pi_{0}(s)+\sum_{l=2}^{\infty} \pi_{i}^{(l)}(s) \sigma_{i}^{(l)}(s-) x^{l}}{1+x \pi_{0}(s)+\sum_{l=2}^{K} \pi_{i}^{(l)}(s) \sigma_{i}^{(l)}(s-) x^{l}}\right)\right. \\
\left.-\sum_{l=K+1}^{\infty} \pi_{i}^{(l)}(s) \sigma_{i}^{(l)}(s-) x^{l}\right)\left.\lambda_{i}(s) \eta(\mathrm{d} x) \mathrm{d} s\right|^{2}
\end{gathered}
$$

tend to 0 as $K \rightarrow \infty$. Indeed, the convergence of Equation (38) follows directly from our assumptions: from the triangle inequality and the inequality (Fechner 2008)

$$
2\left|\exp \left(a_{1}\right)-\exp \left(a_{2}\right)\right| \leq\left|a_{1}-a_{2}\right|\left|\exp \left(a_{1}\right)+\exp \left(a_{2}\right)\right|, a_{1}, a_{2} \in \mathbb{R} .
$$

Then, we get

$$
\mathbb{E}\left|R_{\pi}(t)-R_{\pi}^{K}(t)\right|^{2} \leq \mathbb{E}\left|\log \frac{R_{\pi}(t)}{R_{\pi}^{K}(t)}\right|^{2}\left(\mathbb{E}\left|R_{\pi}(t)\right|^{2}+\mathbb{E}\left|R_{\pi}^{K}(t)\right|^{2}\right)<\infty
$$

and we can use the following equation

$$
\begin{aligned}
\log \frac{R_{\pi}(t)}{R_{\pi}^{K}(t)}= & \log \frac{R_{\pi}(0)}{R_{\pi}^{K}(0)}+\left[\int _ { 0 } ^ { t } \left(\sum_{k=K+1}^{\infty} \pi^{(k)}(s)\left(\mu^{(k)}(s)-r(s-)\right)\right.\right. \\
& \left.+\sum_{i=1}^{N} \sum_{l=K+1}^{\infty} \pi_{i}^{(l)}(s)\left(\mu_{i}^{(l)}(s)-r(s-)\right)-\frac{1}{2} \pi_{0}^{2}(s) \sigma_{0}^{2}(s-)\right) \mathrm{d} s
\end{aligned}
$$




$$
\begin{aligned}
& +\int_{0}^{t} \int_{\mathbb{R}} \log \left(1+\pi_{0}(s) \gamma(s-, x)+\sum_{k=2}^{\infty} \pi^{(k)}(s) \sigma^{(k)}(s-) \gamma^{k}(s-, x)\right) \bar{\Pi}(\mathrm{d} s, \mathrm{~d} x) \\
& -\int_{0}^{t} \int_{\mathbb{R}} \log \left(1+\pi_{0}(s) \gamma(s-, x)+\sum_{k=2}^{K} \pi^{(k)}(s) \sigma^{(k)}(s-) \gamma^{k}(s-, x)\right) \bar{\Pi}(\mathrm{d} s, \mathrm{~d} x) \\
& +\int_{0}^{t} \int_{\mathbb{R}}\left(\log \left(1+\pi_{0}(s) \gamma(s-, x)+\sum_{k=2}^{\infty} \pi^{(k)}(s) \sigma^{(k)}(s-) \gamma^{k}(s-, x)\right)\right. \\
& \left.-\sum_{k=K+1}^{\infty} \pi^{(k)}(s) \sigma^{(k)}(s-) \gamma^{k}(s-, x)\right) v(\mathrm{~d} x) \mathrm{d} s \\
& -\int_{0}^{t} \int_{\mathbb{R}} \log \left(1+\pi_{0}(s) \gamma(s-, x)+\sum_{k=2}^{K} \pi^{(k)}(s) \sigma^{(k)}(s-) \gamma^{k}(s-, x)\right) v(\mathrm{~d} x) \mathrm{d} s \\
& +\sum_{i=1}^{N} \int_{0}^{t} \int_{\mathbb{R}} \log \left(1+x \pi_{0}(s)+\sum_{l=1}^{\infty} \pi_{i}^{(l)}(s) \sigma_{i}^{(l)}(s-) x^{l}\right) \bar{\Pi}_{U}^{i}(\mathrm{~d} s, \mathrm{~d} x) \\
& -\sum_{i=1}^{N} \int_{0}^{t} \int_{\mathbb{R}} \log \left(1+x \pi_{0}(s)+\sum_{l=1}^{K} \pi_{i}^{(l)}(s) \sigma_{i}^{(l)}(s-) x^{l}\right) \bar{\Pi}_{U}^{i}(\mathrm{~d} s, \mathrm{~d} x) \\
& +\sum_{i=1}^{N} \int_{0}^{t} \int_{\mathbb{R}}\left(\log \left(1+x \pi_{0}(s)+\sum_{l=1}^{\infty} \pi_{i}^{(l)}(s) \sigma_{i}^{(l)}(s-) x^{l}\right)\right. \\
& \left.-\sum_{l=K+1}^{\infty} \pi_{i}^{(l)}(s) \sigma_{i}^{(l)}(s-) x^{l}\right) \lambda_{i}(s) \eta(\mathrm{d} x) \mathrm{d} s \\
& \left.-\sum_{i=1}^{N} \int_{0}^{t} \int_{\mathbb{R}} \log \left(1+x \pi_{0}(s)+\sum_{l=1}^{K} \pi_{i}^{(l)}(s) \sigma_{i}^{(l)}(s-) x^{l}\right) \lambda_{i}(s) \eta(\mathrm{d} x) \mathrm{d} s\right]
\end{aligned}
$$

Let $U$ denote a utility function of the investor, which is strictly increasing, strictly concave and twice differentiable, that is, $U^{\prime}>0$ and $U^{\prime \prime}<0$.

For each $(t, z) \in \mathbb{T} \times \mathbb{R}^{+}$and each $i=1, \ldots, N$ we define

$$
V^{\pi}\left(t, z, \mathbf{e}_{i}\right):=\mathbb{E}_{t, z, i}\left[U\left(R_{\pi}(T)\right)\right],
$$

where $\mathbb{E}_{t, z, i}$ is the conditional expectation given $R_{\pi}(0)=z$ and $J(t)=\mathbf{e}_{i}$ under $\mathbb{P}$.

The expectation above is understood in the limiting sense, that is, we limit the set of admissible strategies $\mathcal{A}$ to the strategies $\pi$ such that $\lim _{K \rightarrow \infty} \mathbb{E}_{t, z, i}\left[U\left(R_{\pi}^{K}(T)\right)\right]$ exists and is finite. In other words,

$$
\lim _{K \rightarrow \infty} \mathbb{E}_{t, z, i}\left[U\left(R_{\pi}^{K}(T)\right)\right]=\mathbb{E}_{t, z, i}\left[U\left(R_{\pi}(T)\right)\right]<\infty .
$$

Then, the value function of the investor's portfolio selection problem is defined by

$$
V\left(t, z, \mathbf{e}_{i}\right):=\sup _{\pi \in \mathcal{A}} V^{\pi}\left(t, z, \mathbf{e}_{i}\right)=\sup _{\pi \in \mathcal{A}} \mathbb{E}_{t, z, i}\left[U\left(R_{\pi}(T)\right)\right] .
$$

Lemma 1. Under the assumption in Equation (38), Equation (39) holds true.

The proof of this Lemma is given in Appendix A.

Our main goal is to identify the value function given in Equation (40). In what follows, we consider two risk-averse utility functions, namely, the logarithmic utility and the power utility. 


\subsection{Logarithmic Utility}

In this subsection, we derive the optimal portfolio strategy in the case of a logarithmic utility function of wealth, namely

$$
U(z)=\log (z) .
$$

Recall that in $\mathcal{A}$ we consider only the strategies for which

$$
\lim _{K \rightarrow \infty} \mathbb{E}_{t, z, i}\left[\log R_{\pi}^{K}(T)\right]=\mathbb{E}_{t, z, i}\left[\log R_{\pi}(T)\right]<\infty .
$$

Theorem 5. Assume that there exists a solution

$$
\pi^{\star}(t):=\left(\pi_{0}^{\star}(t), \pi_{1}^{\star}(t), \ldots, \pi_{N}^{\star}(t), \pi^{(2) \star}(t), \pi^{(3) \star}(t), \ldots, \pi_{1}^{(1) \star}(t), \pi_{2}^{(1) \star}(t), \ldots\right)
$$

of the following system of equations (for $i, j=1, \ldots, N, k \geq 2$ and $l \geq 1$ ):

$$
\begin{aligned}
r(t-)-\mu_{0}(t-)= & \pi_{0}^{\star}(t) \sigma_{0}^{2}(t-)+\sum_{i=1}^{N} \frac{r(t-)-\mu_{i}^{(1)}(t-)}{\sigma_{i}^{(1)}(t-)}+\int_{\mathbb{R}} \gamma(t-, x)\left(\left(1+\pi_{0}^{\star}(t) \gamma(t-, x)\right.\right. \\
& \left.\left.+\sum_{k=2}^{\infty} \pi^{(k) \star}(t) \sigma^{(k)}(t-) \gamma^{k}(t-, x)\right)^{-1}-1\right) v(\mathrm{~d} x), \\
\pi_{j}^{\star}(t)= & \frac{\mu_{j}(t-)-r(t-)}{\left(r(t-)-\mu_{j}(t-)\right) \sigma_{j}(t-)+\lambda_{j}(t) \sigma_{j}^{2}(t-)}, \\
\frac{r(t-)-\mu^{(k)}(t-)}{\sigma^{(k)}(t-)}= & \int_{\mathbb{R}} \gamma^{k}(t-, x)\left(\left(1+\pi_{0}^{\star}(t) \gamma(t-, x)\right.\right. \\
\frac{r(t-)-\mu_{i}^{(l)}(t-)}{\sigma_{i}^{(l)}(t-)}= & \int_{\mathbb{R}} x^{l}\left(\left(1+x \pi_{0}^{\star}(t)+\sum_{l=1}^{\infty} \pi_{i}^{(l) \star}(t) \sigma_{i}^{(l)}(t-) x^{l}\right)^{-1}-1\right) \lambda_{i}(t) \eta(\mathrm{d} x),
\end{aligned}
$$

which belongs to $\mathcal{A}$, that is, in particular, satisfies Equations (38) and (41). Then, the optimal portfolio strategy for the portfolio selection problem in Equation (40) with logarithmic utility function of wealth is one of those solutions.

Proof. The conditional expectation of the logarithm of the wealth process has the following form (for $t \in[0, T)$ ):

$$
\begin{aligned}
\mathbb{E}_{t, z, i}\left[\log R_{\pi}(T)\right]=\log R_{\pi}(t)+\mathbb{E}_{t, z, i} \int_{t}^{T}\left[r(s-)+\sum_{j=0}^{N} \pi_{j}(s)\left(\mu_{j}(s-)-r(s-)\right)\right. \\
+\sum_{k=2}^{\infty} \pi^{(k)}(s)\left(\mu^{(k)}(s)-r(s-)\right)+\sum_{i=0}^{N} \sum_{l=1}^{\infty} \pi_{i}^{(l)}(s)\left(\mu_{i}^{(l)}(s)-r(s-)\right)-\frac{1}{2} \pi_{0}^{2}(s) \sigma_{0}^{2}(s-) \\
+\sum_{j=1}^{N}\left(\log \left(1+\pi_{j}(s) \sigma_{j}(s-)\right)-\pi_{j}(s) \sigma_{j}(s-)\right) \lambda_{j}(s) \\
+\int_{\mathbb{R}}\left(\log \left(1+\pi_{0}(s) \gamma(s-, x)+\sum_{k=2}^{\infty} \pi^{(k)}(s) \sigma^{(k)}(s-) \gamma^{k}(s-, x)\right)\right. \\
\left.-\pi_{0}(s) \gamma(s-, x)-\sum_{k=2}^{\infty} \pi^{(k)}(s) \sigma^{(k)}(s-) \gamma^{k}(s-, x)\right) v(\mathrm{~d} x)
\end{aligned}
$$




$$
\left.+\sum_{i=1}^{N} \int_{\mathbb{R}}\left(\log \left(1+x \pi_{0}(s)+\sum_{l=1}^{\infty} \pi_{i}^{(l)}(s) \sigma_{i}^{(l)}(s-) x^{l}\right)-x \pi_{0}(s)-\sum_{l=1}^{\infty} \pi_{i}^{(l)}(s) \sigma_{i}^{(l)}(s-) x^{l}\right) \lambda_{i}(s) \eta(\mathrm{d} x)\right] \mathrm{d} s .
$$

Therefore, the optimal value function $V$ can be written as

$$
V\left(t, z, \mathbf{e}_{i}\right)=\log (z)+\sup _{\pi \in \mathcal{A}} h_{\pi}\left(t, \mathbf{e}_{i}\right)
$$

where

$$
h_{\pi}\left(t, \mathbf{e}_{i}\right):=\mathbb{E}_{t, z, i} \int_{t}^{T} F\left(\pi_{0}(s), \pi_{1}(s), \ldots, \pi_{N}(s), \pi^{(2)}(s), \ldots, \pi_{1}^{(1)}(s), \ldots\right) \mathrm{d} s
$$

for

$$
\begin{aligned}
& F\left(\pi_{0}(s), \pi_{1}(s), \ldots, \pi_{N}(s), \pi^{(2)}(s), \ldots, \pi_{1}^{(1)}(s), \ldots\right):=r(s-)+\sum_{j=0}^{N} \pi_{j}(s)\left(\mu_{j}(s-)-r(s-)\right) \\
& \quad+\sum_{k=2}^{\infty} \pi^{(k)}(s)\left(\mu^{(k)}(s)-r(s-)\right)+\sum_{i=0}^{N} \sum_{l=1}^{\infty} \pi_{i}^{(l)}(s)\left(\mu_{i}^{(l)}(s)-r(s-)\right)-\frac{1}{2} \pi_{0}^{2}(s) \sigma_{0}^{2}(s-) \\
& \quad+\sum_{j=1}^{N}\left(\log \left(1+\pi_{j}(s) \sigma_{j}(s-)\right)-\pi_{j}(s) \sigma_{j}(s-)\right) \lambda_{j}(s) \\
& \quad+\int_{\mathbb{R}}\left(\log \left(1+\pi_{0}(s) \gamma(s-, x)+\sum_{k=2}^{\infty} \pi^{(k)}(s) \sigma^{(k)}(s-) \gamma^{k}(s-, x)\right)\right. \\
& \left.\quad-\pi_{0}(s) \gamma(s-, x)-\sum_{k=2}^{\infty} \pi^{(k)}(s) \sigma^{(k)}(s-) \gamma^{k}(s-, x)\right) v(\mathrm{~d} x) \\
& \quad+\sum_{i=1}^{N} \int_{\mathbb{R}}\left(\log \left(1+x \pi_{0}(s)+\sum_{l=1}^{\infty} \pi_{i}^{(l)}(s) \sigma_{i}^{(l)}(s-) x^{l}\right)-x \pi_{0}(s)-\sum_{l=1}^{\infty} \pi_{i}^{(l)}(s) \sigma_{i}^{(l)}(s-) x^{l}\right) \lambda_{i}(s) \eta(\mathrm{d} x) .
\end{aligned}
$$

Thus, to determine the optimal portfolio strategy, it is sufficient to maximize $F$. Indeed, the maximization of the function $F\left(\pi_{0}(s), \pi_{1}(s), \ldots, \pi_{N}(s), \pi^{(2)}(s), \ldots, \pi_{1}^{(1)}(s), \ldots\right)$ at each time point $s \in[0, T]$ maximizes the integral of $F$ on $[0, T]$. By direct differentiation with respect to $\pi_{0}, \pi_{j}, \pi^{(k)}, \pi_{i}^{(l)}$ we obtain conditions (Equation (42)) that the optimal strategies have to satisfy. Observe that, from Equation (9), the integrals

$$
\begin{aligned}
& \int_{\mathbb{R}}\left(\log \left(1+\pi_{0}(s) \gamma(s-, x)+\sum_{k=2}^{\infty} \pi^{(k)}(s) \sigma^{(k)}(s-) \gamma^{k}(s-, x)\right)-\pi_{0}(s) \gamma(s-, x)\right. \\
& \left.-\sum_{k=2}^{\infty} \pi^{(k)}(s) \sigma^{(k)}(s-) \gamma^{k}(s-, x)\right) v(\mathrm{~d} x)
\end{aligned}
$$

and (for $i=1, \ldots, N)$

$$
\int_{\mathbb{R}}\left(\log \left(1+x \pi_{0}(s)+\sum_{l=1}^{\infty} \pi_{i}^{(l)}(s) \sigma_{i}^{(l)}(s-) x^{l}\right)-x \pi_{0}(s)-\sum_{l=1}^{\infty} \pi_{i}^{(l)}(s) \sigma_{i}^{(l)}(s-) x^{l}\right) \lambda_{i}(s) \eta(\mathrm{d} x)
$$

are well-defined. Hence, by the Leibniz integral rule, we can interchange the above mentioned derivatives and the integrals.

Remark 5. We have not been able to prove that a solution of the system in Equation (42) exists and is unique. On a complete Itô-Markov additive market, we have an infinite number of assets, so the optimal portfolio strategy $\pi^{\star}$ is an infinite dimensional vector. The value function in Equation (40) is understood in the limiting sense and therefore numerically it can be approximated by the finite strategy counterpart. In the case of finite dimensional approximations by Kramkov and Schachermayer (1999, Thm. 2.2), the optimal wealth process exists and is 
unique. Under additional conditions, such as absence of redundant assets, the optimal strategy exists and is unique.

\subsection{Power Utility}

In this subsection, we derive the optimal portfolio strategy in the case of the power utility function, namely

$$
U(z)=z^{\alpha} \quad \text { for } \alpha \in(0,1) .
$$

We assume that, for each $\pi \in \mathcal{A}$,

$$
\lim _{K \rightarrow \infty} \mathbb{E}_{t, z, i}\left(R_{\pi}^{K}(T)\right)^{\alpha}=\mathbb{E}_{t, z, i}\left(R_{\pi}(T)\right)^{\alpha}<\infty .
$$

Theorem 6. Assume that there exists a solution

$$
\pi^{\star}(t):=\left(\pi_{0}^{\star}(t), \pi_{1}^{\star}(t), \ldots, \pi_{N}^{\star}(t), \pi^{(2) \star}(t), \pi^{(3) \star}(t), \ldots, \pi_{1}^{(1) \star}(t), \pi_{2}^{(1) \star}(t), \ldots\right)
$$

of the following system of equations (for $i, j=1, \ldots, N, k \geq 2$ and $l \geq 1$ ):

$$
\begin{aligned}
r(t-)-\mu_{0}(t)= & (\alpha-1) \pi_{0}^{\star}(t) \sigma_{0}^{2}(t-)+\sum_{i=1}^{N} \frac{\mu_{i}^{(1)}(t-)-r(t-)}{\sigma_{i}^{(1)}(t-)}+\int_{\mathbb{R}} \gamma(t-, x)\left(\left(1+\pi_{0}^{\star}(t) \gamma(t-, x)\right.\right. \\
& \left.\left.+\sum_{k=2}^{\infty} \pi^{(k) \star}(t) \sigma^{(k)}(t-) \gamma^{k}(t-, x)\right)^{\alpha-1}-1\right) v(\mathrm{~d} x), \\
\pi_{j}^{\star}(t)= & \frac{\left(1-\frac{\mu_{j}(t-)-r(t-)}{\lambda_{i}(t) \sigma_{j}(t-)}\right)^{\frac{1}{\alpha-1}}-1}{\sigma_{j}(t-)}, \\
r(t-)-\mu^{(k)}(t)= & \int_{\mathbb{R}} \sigma^{(k)}(t-) \gamma^{k}(t-, x)\left(\left(1+\pi_{0}^{\star}(t) \gamma(t-, x)\right.\right. \\
& \left.\left.+\sum_{k=2}^{\infty} \pi^{(k) \star}(t) \sigma^{(k)}(t-) \gamma^{k}(t-, x)\right)^{\alpha-1}-1\right) v(\mathrm{~d} x), \\
r(t-)-\mu_{i}^{(l)}(t)= & \int_{\mathbb{R}} \sigma_{i}^{(l)}(t-) x^{l}\left(\left(1+x \pi_{0}^{\star}(t)+\sum_{l=1}^{\infty} \pi_{i}^{\star(l)}(t) x^{l} \sigma_{i}^{(l)}(t-)\right)^{\alpha-1}-1\right) \lambda_{i}(t) \eta(\mathrm{d} x) \mathrm{d} t,
\end{aligned}
$$

which belongs to $\mathcal{A}$, that is, in particular, it satisfies Equations (38) and (43). Then, the optimal portfolio strategy for the portfolio selection problem in Equation (40) with power utility function of wealth is one of those solutions.

Proof. From Itô's formula (see Protter (2005, Thm. 32)) for the power utility function of wealth, we obtain (for $s \in[t, T]$ and $t \in[0, T]$ )

$$
\begin{aligned}
& \left(R_{\pi}(T)\right)^{\alpha}-\left(R_{\pi}(t)\right)^{\alpha}=\int_{t}^{T} \alpha\left(R_{\pi}(s)\right)^{\alpha}\left(r(s-)+\sum_{j=0}^{N} \pi_{j}(s)\left(\mu_{j}(s-)-r(s-)\right)+\sum_{k=2}^{\infty} \pi^{(k)}(s)\left(\mu^{(k)}(s)-r(s-)\right)\right. \\
& \left.\quad+\sum_{i=0}^{N} \sum_{l=1}^{\infty} \pi_{i}^{(l)}(s)\left(\mu_{i}^{(l)}(s)-r(s-)\right)\right) \mathrm{d} s+\int_{t}^{T} \alpha\left(R_{\pi}(s)\right)^{\alpha} \pi_{0}(s) \sigma_{0}(s-) \mathrm{d} W(s) \\
& \quad+\int_{t}^{T} \frac{1}{2} \alpha(\alpha-1)\left(R_{\pi}(s)\right)^{\alpha} \pi_{0}^{2}(s) \sigma_{0}^{2}(s-) \mathrm{d} s+\sum_{j=1}^{N} \int_{t}^{T}\left(\left(R_{\pi}(s)+R_{\pi}(s) \pi_{j}(s) \sigma_{j}(s-)\right)^{\alpha}-\left(R_{\pi}(s)\right)^{\alpha}\right) \mathrm{d} \bar{\Phi}_{j}(s) \\
& \quad+\sum_{j=1}^{N} \int_{t}^{T}\left(\left(R_{\pi}(s)+R_{\pi}(s) \pi_{j}(s) \sigma_{j}(s-)\right)^{\alpha}-\left(R_{\pi}(s)\right)^{\alpha}-\alpha\left(R_{\pi}(s)\right)^{\alpha} \pi_{j}(s) \sigma_{j}(s-)\right) \lambda_{j}(s) \mathrm{d} s
\end{aligned}
$$




$$
\begin{aligned}
& +\int_{t}^{T} \int_{\mathbb{R}}\left(\left(R_{\pi}(s-)+R_{\pi}(s)\left(\pi_{0}(s) \gamma(s-, x)+\sum_{k=2}^{\infty} \pi^{(k)}(s) \sigma^{(k)}(s-) \gamma^{k}(s-, x)\right)\right)^{\alpha}\right. \\
& \left.-\left(R_{\pi}(s-)\right)^{\alpha}-\alpha\left(R_{\pi}(s-)\right)^{\alpha}\left(\pi_{0}(s) \gamma(s-, x)+\sum_{k=2}^{\infty} \pi^{(k)}(s) \sigma^{(k)}(s-) \gamma^{k}(s-, x)\right)\right) v(\mathrm{~d} x) \mathrm{d} s \\
& +\int_{t}^{T} \int_{\mathbb{R}}\left(\left(R_{\pi}(s-)+R_{\pi}(s)\left(\pi_{0}(s) \gamma(s-, x)+\sum_{k=2}^{\infty} \pi^{(k)}(s) \sigma^{(k)}(s-) \gamma^{k}(s-, x)\right)\right)^{\alpha}-\left(R_{\pi}(s-)\right)^{\alpha}\right) \bar{\Pi}(\mathrm{d} s, \mathrm{~d} x) \\
& +\sum_{i=1}^{N} \int_{t}^{T} \int_{\mathbb{R}}\left(\left(R_{\pi}(s-)+R_{\pi}(s)\left(x \pi_{0}(s)+\sum_{l=1}^{\infty} x^{l} \pi_{i}^{(l)}(s) \sigma_{i}^{(l)}(s-)\right)\right)^{\alpha}-\left(R_{\pi}(s-)\right)^{\alpha}\right. \\
& \left.-\alpha\left(R_{\pi}(s-)\right)^{\alpha}\left(x \pi_{0}(s)+\sum_{l=1}^{\infty} x^{l} \pi_{i}^{(l)}(s) \sigma_{i}^{(l)}(s-)\right)\right)^{\lambda}(s) \eta(\mathrm{d} x) \mathrm{d} s \\
& +\sum_{i=1}^{N} \int_{t}^{T} \int_{\mathbb{R}}\left(\left(R_{\pi}(s-)+R_{\pi}(s)\left(x \pi_{0}(s)+\sum_{l=1}^{\infty} x^{l} \pi_{i}^{(l)}(s) \sigma_{i}^{(l)}(s-)\right)\right)^{\alpha}-\left(R_{\pi}(s-)\right)^{\alpha}\right) \bar{\Pi}_{U}^{i}(\mathrm{~d} s, \mathrm{~d} x) .
\end{aligned}
$$

From this and Equation (40), the value function is given by

$$
\begin{aligned}
V\left(t, z, \mathbf{e}_{i}\right)= & z^{\alpha}+\sup _{\pi \in \mathcal{A}} \mathbb{E}_{t, z, i} \int_{t}^{T} z^{\alpha}\left[\alpha \left(r(s-)+\sum_{j=0}^{N} \pi_{j}(s)\left(\mu_{j}(s-)-r(s-)\right)+\sum_{k=2}^{\infty} \pi^{(k)}(s)\left(\mu^{(k)}(s)-r(s-)\right)\right.\right. \\
& \left.+\sum_{i=0}^{N} \sum_{l=1}^{\infty} \pi_{i}^{(l)}(s)\left(\mu_{i}^{(l)}(s)-r(s-)\right)+\frac{1}{2}(\alpha-1) \pi_{0}^{2}(s) \sigma_{0}^{2}(s-)\right) \\
& +\sum_{j=1}^{N}\left(\left(1+\pi_{j}(s) \sigma_{j}(s-)\right)^{\alpha}-1-\alpha \pi_{j}(s) \sigma_{j}(s-)\right) \lambda_{j}(s) \\
& +\int_{\mathbb{R}}\left(\left(1+\pi_{0}(s) \gamma(s-, x)+\sum_{k=2}^{\infty} \pi^{(k)}(s) \sigma^{(k)}(s-) \gamma^{k}(s-, x)\right)^{\alpha}\right. \\
& \left.-1-\alpha\left(\pi_{0}(s) \gamma(s-, x)+\sum_{k=2}^{\infty} \pi^{(k)}(s) \sigma^{(k)}(s-) \gamma^{k}(s-, x)\right)\right) v(\mathrm{~d} x) \\
& +\sum_{i=1}^{N} \int_{\mathbb{R}}\left(\left(1+x \pi_{0}(s)+\sum_{l=1}^{\infty} x^{l} \pi_{i}^{(l)}(s) \sigma_{i}^{(l)}(s-)\right)^{\alpha}-1\right. \\
& \left.\left.-\alpha\left(x \pi_{0}(s)+\sum_{l=1}^{\infty} x^{l} \pi_{i}^{(l)}(s) \sigma_{i}^{(l)}(s-)\right)\right) \lambda_{i}(s) \eta(\mathrm{d} x)\right] \mathrm{d} s .
\end{aligned}
$$

By direct differentiation with respect to each strategy, this supremum is attained if the strategies satisfy the system in Equation (44). Observe that, from Equation (9), the integrals

$$
\begin{aligned}
& \int_{\mathbb{R}}\left(\left(1+\pi_{0}(s) \gamma(s-, x)+\sum_{k=2}^{\infty} \pi^{(k)}(s) \sigma^{(k)}(s-) \gamma^{k}(s-, x)\right)^{\alpha}\right. \\
& \left.-1-\alpha\left(\pi_{0}(s) \gamma(s-, x)+\sum_{k=2}^{\infty} \pi^{(k)}(s) \sigma^{(k)}(s-) \gamma^{k}(s-, x)\right)\right) \nu(\mathrm{d} x)
\end{aligned}
$$

and (for $i=1, \ldots, N)$

$$
\int_{\mathbb{R}}\left(\left(1+x \pi_{0}(s)+\sum_{l=1}^{\infty} x^{l} \pi_{i}^{(l)}(s) \sigma_{i}^{(l)}(s-)\right)^{\alpha}-1-\alpha\left(x \pi_{0}(s)+\sum_{l=1}^{\infty} x^{l} \pi_{i}^{(l)}(s) \sigma_{i}^{(l)}(s-)\right)\right) \lambda_{i}(s) \eta(\mathrm{d} x)
$$

are well-defined. Hence, by the Leibniz integral rule, we can interchange the derivatives and integrals. 
Remark 6. We have not been able to prove that a solution of Equation (44) exists and is unique. However, if we consider a finite market, then this solution exists and is unique and must be the optimal portfolio by Kramkov and Schachermayer (1999, Thm. 2.2). In $\pi_{j}^{*}(t)$, one can observe the dependence of this strategy on Sharpe ratio.

Remark 7. If the prices of assets in the Black-Scholes-Merton market are described by processes without jumps (that is, $\bar{\Pi}(\mathrm{d} t, \mathrm{~d} x)=0$ and $\left.\bar{\Pi}_{U}^{i}(\mathrm{~d} t, \mathrm{~d} x)=0\right)$, then we obtain closed-form solutions to the optimal portfolio selection problem in Equation (40) for the logarithmic and power utilities (Zhang et al. 2010). In addition, the value function in the primary market is the same as in the enlarged market, while in our market this does not occur.

\section{Optimal Portfolio Selection in the Original Market}

In this section, we find conditions for optimal portfolio strategies in the original market, i.e., in the market with one risk-free asset and one share.

Let $\tilde{\pi}_{0}$ be the proportion of wealth invested in share $S_{0}$ in the original market. Then, the corresponding wealth process, denoted as $R^{\tilde{\pi}_{0}}$, is given by the stochastic differential equation

$$
\begin{aligned}
\frac{\mathrm{d} R_{\tilde{\pi}_{0}}(t)}{R_{\tilde{\pi}_{0}}(t-)}:= & \left(r(t-)+\tilde{\pi}_{0}(t)\left(\mu_{0}(t-)-r(t-)\right)\right) \mathrm{d} t+\tilde{\pi}_{0}(t) \sigma_{0}(t-) \mathrm{d} W(t) \\
& +\tilde{\pi}_{0}(t) \int_{\mathbb{R}} \gamma(t-, x) \bar{\Pi}(\mathrm{d} t, \mathrm{~d} x)+\tilde{\pi}_{0}(t) \sum_{i=1}^{N} \int_{\mathbb{R}} x \bar{\Pi}_{U}^{i}(\mathrm{~d} t, \mathrm{~d} x) .
\end{aligned}
$$

Let $\mathcal{A}_{0}$ be the class of admissible portfolio strategies $\tilde{\pi}_{0}$ such that $\tilde{\pi}_{0}$ is predictable, $\left\{\mathcal{F}_{t}\right\}$-adaptable and satisfies the condition $\int_{t}^{T}\left|\tilde{\pi}_{0}(s)\right|^{2} \mathrm{~d} s<\infty, \mathbb{P}-$ a.s. Similar to the definition of the value function in the enlarged market, we define the value function in the original incomplete market as

$$
V_{0}\left(t, z, \mathbf{e}_{i}\right):=\sup _{\tilde{\pi}_{0} \in \mathcal{A}_{0}} \mathbb{E}_{t, z, i}\left[U\left(R^{\tilde{\pi}_{0}}(T)\right)\right]
$$

We assume $\mathbb{E}_{t, z, i}\left[U\left(R_{\tilde{\pi}_{0}}(T)\right)\right]<\infty$ for $i=1, \ldots, N$.

First, we consider the logarithmic utility function. We have

$$
\begin{aligned}
\mathbb{E}_{t, z, i}\left[\log R_{\tilde{\pi}_{0}}(T)\right]= & \log z+\mathbb{E}_{t, z, i} \int_{t}^{T}\left[r(s-)+\tilde{\pi}_{0}(s)\left(\mu_{0}(s-)-r(s-)\right)-\frac{1}{2} \tilde{\pi}_{0}^{2}(s) \sigma_{0}^{2}(s-)\right. \\
& +\int_{\mathbb{R}}\left(\log \left(1+\tilde{\pi}_{0}(s) \gamma(s-, x)\right)-\tilde{\pi}_{0}(s) \gamma(s-, x)\right) v(\mathrm{~d} x) \\
& \left.+\sum_{i=1}^{N} \int_{\mathbb{R}}\left(\log \left(1+x \tilde{\pi}_{0}(s)\right)-x \tilde{\pi}_{0}(s)\right) \lambda_{i}(s) \eta(\mathrm{d} x)\right] \mathrm{d} s,
\end{aligned}
$$

that is, for the logarithmic utility, the value function $V_{0}$ can be written as follows:

$$
\begin{aligned}
V_{0}\left(t, z, \mathbf{e}_{i}\right)= & \log z+\sup _{\tilde{\pi}_{0} \in \mathcal{A}_{0}} \mathbb{E}_{t, z, i} \int_{t}^{T}\left[r(s-)+\tilde{\pi}_{0}(s)\left(\mu_{0}(s-)-r(s-)\right)-\frac{1}{2} \tilde{\pi}_{0}^{2}(s) \sigma_{0}^{2}(s-)\right. \\
& +\int_{\mathbb{R}}\left(\log \left(1+\tilde{\pi}_{0}(s) \gamma(s-, x)\right)-\tilde{\pi}_{0}(s) \gamma(s-, x)\right) v(\mathrm{~d} x) \\
& \left.+\sum_{i=1}^{N} \int_{\mathbb{R}}\left(\log \left(1+x \tilde{\pi}_{0}(s)\right)-x \tilde{\pi}_{0}(s)\right) \lambda_{i}(s) \eta(\mathrm{d} x)\right] \mathrm{d} s .
\end{aligned}
$$

From Equation (9), the integrals

$$
\int_{\mathbb{R}}\left(\log \left(1+\tilde{\pi}_{0}(s) \gamma(s-, x)\right)-\tilde{\pi}_{0}(s) \gamma(s-, x)\right) v(\mathrm{~d} x)
$$


and (for $i=1, \ldots, N)$

$$
\int_{\mathbb{R}}\left(\log \left(1+x \tilde{\pi}_{0}(s)\right)-x \tilde{\pi}_{0}(s)\right) \lambda_{i}(s) \eta(\mathrm{d} x)
$$

are well-defined. Hence, by the Leibniz integral rule, we can differentiate the above integrals with respect to $\tilde{\pi}_{0}$. The above supremum is attained if $\tilde{\pi}_{0}$ solves the equation

$$
\begin{aligned}
& \mu_{0}(s-)-r(s)-\sigma_{0}^{2}(s-) \tilde{\pi}_{0}(s)+\int_{\mathbb{R}}\left(\frac{\gamma(s-, x)}{1+\tilde{\pi}_{0}(s) \gamma(s-, x)}-\gamma(s-, x)\right) v(\mathrm{~d} x) \\
& +\sum_{i=1}^{N} \int_{\mathbb{R}}\left(\frac{x}{1+x \tilde{\pi}_{0}(s)}-x\right) \lambda_{i}(s) \eta(\mathrm{d} x)=0 .
\end{aligned}
$$

In the case of the power utility, the value function $V_{0}$ can be written as

$$
\begin{aligned}
V_{0}\left(t, z, \mathbf{e}_{i}\right)= & z^{\alpha}+\sup _{\tilde{\pi}_{0} \in \mathcal{A}_{0}} \mathbb{E}_{t, z, i} \int_{t}^{T}\left[\alpha z^{\alpha}\left(r(s-)+\tilde{\pi}_{0}(s)\left(\mu_{0}(s-)-r(s-)\right)+\frac{1}{2}(\alpha-1) \tilde{\pi}_{0}^{2}(s) \sigma_{0}^{2}(s-)\right)\right. \\
& +\int_{\mathbb{R}} z^{\alpha}\left(\left(1+\tilde{\pi}_{0}(s) \gamma(s-, x)\right)^{\alpha}-1-\alpha \tilde{\pi}_{0}(s) \gamma(s-, x)\right) v(\mathrm{~d} x) \\
& \left.+\sum_{i=1}^{N} \int_{\mathbb{R}} z^{\alpha}\left(\left(1+x \tilde{\pi}_{0}(s)\right)^{\alpha}-1-\alpha x \tilde{\pi}_{0}(s)\right) \lambda_{i}(s) \eta(\mathrm{d} x)\right] \mathrm{d} s .
\end{aligned}
$$

Note that, from Equation (9), the integrals

$$
\int_{\mathbb{R}} z^{\alpha}\left(\left(1+\tilde{\pi}_{0}(s) \gamma(s-, x)\right)^{\alpha}-1-\alpha \tilde{\pi}_{0}(s) \gamma(s-, x)\right) v(\mathrm{~d} x)
$$

and (for $i=1, \ldots, N)$

$$
\int_{\mathbb{R}} z^{\alpha}\left(\left(1+x \tilde{\pi}_{0}(s)\right)^{\alpha}-1-\alpha x \tilde{\pi}_{0}(s)\right) \lambda_{i}(s) \eta(\mathrm{d} x)
$$

are well-defined. By direct differentiation in Equation (47) with respect to $\tilde{\pi}_{0}$, we get

$$
\begin{aligned}
& \mu_{0}(s-)-r(s)-(\alpha-1) \sigma_{0}^{2}(s-) \tilde{\pi}_{0}(s)+\int_{\mathbb{R}}\left(\gamma(s-, x)\left(1+\tilde{\pi}_{0}(s) \gamma(s-, x)\right)^{\alpha-1}-\gamma(s-, x)\right) v(\mathrm{~d} x) \\
& +\sum_{i=1}^{N} \int_{\mathbb{R}}\left(x\left(1+x \tilde{\pi}_{0}(s)\right)^{\alpha-1}-x\right) \lambda_{i}(s) \eta(\mathrm{d} x)=0 .
\end{aligned}
$$

Lemma 2. The solutions of Equations (46) and (47) are optimal strategies for the portfolio selection problem in Equation (45) for the logarithmic and power utilities, respectively.

Proof. We prove that solutions of Equations (46) and (47) are optimal portfolio strategies; their existence was proved by Kramkov and Schachermayer (1999). Let $\tilde{\pi}_{0}^{\varepsilon}:=\tilde{\pi}_{0}+\varepsilon$ be a perturbed portfolio strategy for $\varepsilon>0$.

We define the value function $V_{0}^{\varepsilon}$ related to the strategy $\tilde{\pi}_{0}^{\varepsilon}$ (Fouque et al. 2017a, 2017b; Mokkhavesa and Atkinson 2002) as follows:

$$
V_{0}^{\varepsilon}\left(t, z, \mathbf{e}_{i}\right):=\sup _{\tilde{\pi}_{0}^{\varepsilon} \in \mathcal{A}_{0}} \mathbb{E}_{t, z, i}\left[U\left(R_{\tilde{\pi}_{0}^{\varepsilon}}(T)\right)\right]
$$


In the case of the logarithmic utility,

$$
\begin{aligned}
V_{0}^{\varepsilon}\left(t, z, \mathbf{e}_{i}\right)= & \log z+\sup _{\tilde{\pi}_{0} \in \mathcal{A}_{0}} \mathbb{E}_{t, z, i} \int_{t}^{T}\left[r(s-)+\left(\tilde{\pi}_{0}(s)+\varepsilon\right)\left(\mu_{0}(s-)-r(s-)\right)-\frac{1}{2}\left(\tilde{\pi}_{0}(s)+\varepsilon\right)^{2} \sigma_{0}^{2}(s-)\right. \\
& +\int_{\mathbb{R}}\left(\log \left(1+\left(\tilde{\pi}_{0}(s)+\varepsilon\right) \gamma(s-, x)\right)-\left(\tilde{\pi}_{0}(s)+\varepsilon\right) \gamma(s-, x)\right) v(\mathrm{~d} x) \\
& \left.+\sum_{i=1}^{N} \int_{\mathbb{R}}\left(\log \left(1+x\left(\tilde{\pi}_{0}(s)+\varepsilon\right)\right)-x\left(\tilde{\pi}_{0}(s)+\varepsilon\right)\right) \lambda_{i}(s) \eta(\mathrm{d} x)\right] \mathrm{d} s,
\end{aligned}
$$

and for the power utility,

$$
\begin{aligned}
V_{0}^{\varepsilon}\left(t, z, \mathbf{e}_{i}\right)= & z^{\alpha}+\sup _{\tilde{\pi}_{0} \in \mathcal{A}_{0}} \mathbb{E}_{t, z, i} \int_{t}^{T}\left[\alpha z ^ { \alpha } \left(r(s-)+\left(\tilde{\pi}_{0}(s)+\varepsilon\right)\left(\mu_{0}(s-)-r(s-)\right)\right.\right. \\
& \left.+\frac{1}{2}(\alpha-1)\left(\tilde{\pi}_{0}(s)+\varepsilon\right)^{2} \sigma_{0}^{2}(s-)\right) \\
& +\int_{\mathbb{R}} z^{\alpha}\left(\left(1+\left(\tilde{\pi}_{0}(s)+\varepsilon\right) \gamma(s-, x)\right)^{\alpha}-1-\alpha\left(\tilde{\pi}_{0}(s)+\varepsilon\right) \gamma(s-, x)\right) v(\mathrm{~d} x) \\
& \left.+\sum_{i=1}^{N} \int_{\mathbb{R}} z^{\alpha}\left(\left(1+x\left(\tilde{\pi}_{0}(s)+\varepsilon\right)\right)^{\alpha}-1-\alpha x\left(\tilde{\pi}_{0}(s)+\varepsilon\right)\right) \lambda_{i}(s) \eta(\mathrm{d} x)\right] \mathrm{d} s .
\end{aligned}
$$

Note that $\tilde{\pi}_{0}$ is a portfolio strategy that maximizes the value function, so $\left.\frac{\partial}{\partial \varepsilon} V_{0}^{\varepsilon}\right|_{\varepsilon=0}=0$. Calculating this derivative for $V_{0}^{\varepsilon}$ in both cases, we get Equations (46) and (47).

Thus, the optimal portfolio strategies solve these equations. The existence and uniqueness of the optimal strategies follows from Kramkov and Schachermayer (1999). In that paper, the main assumption concerns the utility function, which has to have asymptotic elasticity strictly less than 1 , that is,

$$
\varlimsup_{z \rightarrow \infty} \frac{z U^{\prime}(z)}{U(z)}<1
$$

Note that the power and logarithmic utilities satisfy this condition.

Remark 8. In a general semi-martingale market model, Goll and Kallsen $(2000,2003)$ obtained the optimal solution explicitly in terms of semi-martingale characteristics of the price process for the logarithmic utility.

Author Contributions: Methodology, Z.P. and Ł.S.; Formal Analysis, A.S., Z.P. and Ł.S.; Investigation, A.S., Z.P. and Ł.S.; Writing—Original Draft Preparation, A.S.; Writing—Review and Editing, A.S., Z.P. and Ł.S.; Visualization, A.S.; Supervision, Z.P. and Ł.S.; Project Administration, Z.P. and Ł.S.; and Funding Acquisition, Z.P.

Funding: This paper was supported by the National Science Centre under the grant 2016/23/B/HS4/00566 (2017-2020).

Acknowledgments: Anna Sulima would like to thank Monique Pontier (Université Paul Sabatier, Toulouse) for plenty of helpful suggestions that improved this paper.

Conflicts of Interest: The authors declare no conflict of interest. The funders had no role in the design of the study; in the collection, analyses, or interpretation of data; in the writing of the manuscript, or in the decision to publish the results. 


\section{Appendix A}

Proof of Lemma 1. We define

$$
U_{M}(z):=U(z) \mathbf{1}_{\{z:|U(z)| \leq M\}} .
$$

The convergence of $R_{\pi}^{K}$ to $R_{\pi}$ in $L^{2}(\Omega, \mathcal{F}, \mathbb{P})$ given in Equation (38) implies the convergence in probability of $R_{\pi}^{K}$ to $R_{\pi}$ as $K \rightarrow \infty$ (see Jacod and Protter (2004, Thm. 17.2)).

Thus, for the bounded and continuous function $U_{M}$ given above, we have

$$
\lim _{K \rightarrow \infty} \mathbb{E}_{t, z, i}\left[U_{M}\left(R_{\pi}^{K}(T)\right)\right]=\mathbb{E}_{t, z, i}\left[U_{M}\left(R_{\pi}(T)\right)\right]
$$

(see Jacod and Protter (2004, Thm. 18.1)). Note that

$$
\mathbb{E}_{t, z, i}\left[U\left(R_{\pi}^{K}(T)\right)\right]=\mathbb{E}_{t, z, i}\left[U_{M}\left(R_{\pi}^{K}(T)\right)\right]+\mathbb{E}_{t, z, i}\left[U\left(R_{\pi}^{K}(T)\right) \mathbf{1}_{\left\{R_{\pi}^{K}(T):\left|U\left(R_{\pi}^{K}(T)\right)\right|>M\right\}}\right] .
$$

From the concavity of $U$, it follows that $U(z) \leq b+c z$ for each $z \geq 0$ and some real $b, c \geq 0$. Thus, the second term on the right-hand side of Equation (A2) satisfies

$$
\mathbb{E}_{t, z, i}\left[U\left(R_{\pi}^{K}(T)\right) \mathbf{1}_{\left\{R_{\pi}^{K}(T):\left|U\left(R_{\pi}^{K}(T)\right)\right|>M\right\}}\right] \leq b+c \mathbb{E}_{t, z, i}\left[R_{\pi}^{K}(T) \mathbf{1}_{\left\{R_{\pi}^{K}(T):\left|U\left(R_{\pi}^{K}(T)\right)\right|>M\right\}}\right] .
$$

Now, we prove that

$$
\lim _{K \rightarrow \infty} \mathbb{E}_{t, z, i}\left[R_{\pi}^{K}(T) \mathbb{I}^{K}(T)\right]=\mathbb{E}_{t, z, i}\left[R_{\pi}(T) \mathbb{I}(T)\right]
$$

where

$$
\mathbb{I}^{K}(T):=\mathbf{1}_{\left\{R_{\pi}^{K}(T):\left|U\left(R_{\pi}^{K}(T)\right)\right|>M\right\}} \text { and } \mathbb{I}(T):=\mathbf{1}_{\left\{R_{\pi}(T):\left|U\left(R_{\pi}(T)\right)\right|>M\right\}} .
$$

Note that

$$
\begin{aligned}
\mathbb{E}_{t, z, i} \mid & R_{\pi}^{K}(T) \mathbb{I}^{K}(T)-R_{\pi}(T) \mathbb{I}(T) \mid \\
\leq & {\left[\mathbb{E}_{t, z, i}\left(R_{\pi}^{K}(T)-R_{\pi}(T)\right)^{2}\right]^{\frac{1}{2}}\left[\mathbb{E}_{t, z, i}(\mathbb{I}(T))\right]^{\frac{1}{2}} } \\
+ & {\left[\mathbb{E}_{t, z, i}\left(R_{\pi}(T)\right)^{2}\right]^{\frac{1}{2}}\left[\mathbb{E}_{t, z, i}\left(\mathbb{I}(T)-\mathbb{I}^{K}(T)\right)^{2}\right]^{\frac{1}{2}} } \\
+ & {\left[\mathbb{E}_{t, z, i}\left(R_{\pi}^{K}(T)\right)^{2}\right]^{\frac{1}{2}}\left[\mathbb{E}_{t, z, i}\left(\mathbb{I}^{K}(T)-\mathbb{I}(T)\right)^{2}\right]^{\frac{1}{2}} } \\
+ & {\left[\mathbb{E}_{t, z, i}\left(R_{\pi}(T)\right)^{2}\right]^{\frac{1}{2}}\left[\mathbb{E}_{t, z, i}\left(\mathbb{I}^{K}(T)-\mathbb{I}(T)\right)^{2}\right]^{\frac{1}{2}} }
\end{aligned}
$$

Indeed, from the triangle inequality, we obtain

$$
\begin{aligned}
& \mathbb{E}_{t, z, i}\left|R_{\pi}^{K}(T) \mathbb{I}^{K}(T)-R_{\pi}(T) \mathbb{I}(T)\right| \\
& \leq \quad \mathbb{E}_{t, z, i}\left|R_{\pi}^{K}(T) \mathbb{I}^{K}(T)-R_{\pi}^{K}(T) \mathbb{I}(T)\right|+\mathbb{E}_{t, z, i}\left|R_{\pi}^{K}(T) \mathbb{I}(T)-R_{\pi}(T) \mathbb{I}^{K}(T)\right| \\
& \quad+\mathbb{E}_{t, z, i}\left|R_{\pi}(T) \mathbb{I}^{K}(T)-R_{\pi}(T) \mathbb{I}(T)\right| .
\end{aligned}
$$

Moreover, using Hölder's inequality, we get

$$
\mathbb{E}_{t, z, i}\left|R_{\pi}^{K}(T) \mathbb{I}^{K}(T)-R_{\pi}^{K}(T) \mathbb{I}(T)\right| \leq\left[\mathbb{E}_{t, z, i}\left(R_{\pi}^{K}(T)\right)^{2}\right]^{\frac{1}{2}}\left[\mathbb{E}_{t, z, i}\left(\mathbb{I}^{K}(T)-\mathbb{I}(T)\right)^{2}\right]^{\frac{1}{2}}
$$


and

$$
\mathbb{E}_{t, z, i}\left|R_{\pi}(T) \mathbb{I}^{K}(T)-R_{\pi}(T) \mathbb{I}(T)\right| \leq\left[\mathbb{E}_{t, z, i}\left(R_{\pi}(T)\right)^{2}\right]^{\frac{1}{2}}\left[\mathbb{E}_{t, z, i}\left(\mathbb{I}^{K}(T)-\mathbb{I}(T)\right)^{2}\right]^{\frac{1}{2}}
$$

Finally, from the triangle inequality and Hölder's inequality, we derive

$$
\begin{aligned}
\mathbb{E}_{t, z, i}\left|R_{\pi}^{K}(T) \mathbb{I}(T)-R_{\pi}(T) \mathbb{I}^{K}(T)\right| \\
\leq \quad\left[\mathbb{E}_{t, z, i}\left(R_{\pi}^{K}(T)-R_{\pi}(T)\right)^{2}\right]^{\frac{1}{2}}\left[\mathbb{E}_{t, z, i}(\mathbb{I}(T))^{\frac{1}{2}}\right. \\
\quad+\left[\mathbb{E}_{t, z, i}\left(R_{\pi}(T)\right)^{2}\right]^{\frac{1}{2}}\left[\mathbb{E}_{t, z, i}\left(\mathbb{I}(T)-\mathbb{I}^{K}(T)\right)^{2}\right]^{\frac{1}{2}} .
\end{aligned}
$$

Combining Equations (A5)-(A8), we get the inequality in Equation (A4).

Now, we prove that

$$
\mathbb{I}^{K} \rightarrow \mathbb{I}
$$

in $L^{2}(\Omega, \mathcal{F}, \mathbb{P})$ as $K \rightarrow \infty$.

First, we verify this convergence in probability. Indeed, for any $\varepsilon>0$, there exists $\delta>0$ such that

$$
\begin{aligned}
\mathbb{P}\left(\left|\mathbb{I}^{K}(T)-\mathbb{I}(T)\right|>\varepsilon\right)= & \mathbb{P}\left(\left\{U\left(R_{\pi}^{K}(T)\right)>M, U\left(R_{\pi}(T)\right)<M\right\} \cup\left\{U\left(R_{\pi}^{K}(T)\right)<M, U\left(R_{\pi}(T)\right)>M\right\}\right) \\
& \leq \mathbb{P}\left(\left|U\left(R_{\pi}^{K}(T)\right)-U\left(R_{\pi}(T)\right)\right|>\delta\right) .
\end{aligned}
$$

The right-hand side of the above inequality tends to zero as $K \rightarrow \infty$, since the convergence in probability of $R_{\pi}^{K}$ to $R_{\pi}$ yields the convergence in probability of $U\left(R_{\pi}^{K}\right)$ to $U\left(R_{\pi}\right)$ (see Jacod and Protter (2004, Thm. 17.5)).

Moreover, we have

$$
\mathbb{I}^{K}(T) \leq 1
$$

thus $\left\{\mathbb{I}^{K}\right\}_{K=2}^{\infty}$ is uniformly integrable and

$$
\lim _{K \rightarrow \infty} \mathbb{I}^{K}(T)=\mathbb{I}(T)
$$

in $L^{2}(\Omega, \mathcal{F}, \mathbb{P})$ (see Gut (2005, Thm. 4.5, p. 216 and Thm. 5.4, p. 221 )). This completes the proof of Equation (A9).

By Equations (38) and (A9), the right-hand side of the inequality in Equation (A4) tends to 0 as $K \rightarrow \infty$. This completes the proof of Equation (A3).

From Equation (A3), it follows that

$$
\varlimsup_{K \rightarrow \infty} \mathbb{E}_{t, z, i}\left[U\left(R_{\pi}^{K}(T)\right) \mathbf{1}_{\left\{R_{\pi}^{K}(T):\left|U\left(R_{\pi}^{K}(T)\right)\right|>M\right\}}\right]
$$

is well-defined. Thus, as $M \rightarrow \infty$, the second term on the right-hand side of Equation (A2) tends to zero. Moreover, the first term converges to $\mathbb{E}_{t, z, i}\left[U\left(R_{\pi}(T)\right)\right]$ by (A1). This completes the proof.

\section{References}

Artzner, Philippe, and David Heath. 1995. Approximate completeness with multiple martingale measures. Mathematical Finance 5: 1-11.

Asmussen, Søren. 2003. Applied Probability and Queues, 2nd ed; Berlin and Heidelberg: Springer.

Asmussen, Søren, and Offer Kella. 2000. Multi-dimensional martingale for Markov additive processes and its applications. Advances in Applied Probability 32: 376-80. [CrossRef] 
Bae, Geum, Woo Chang Kim, and John M. Mulvey. 2014. Dynamic asset allocation for varied financial markets under regime switching framework. European Journal of Operational Research 234: 450-58. [CrossRef]

Barndorff-Nielsen, Ole E., and Neil Shephard. 2003. Realised power variation and stochastic volatility models. Bernoulli 9: 243-65. [CrossRef]

Barndorff-Nielsen, Ole E., and Neil Shephard. 2004. Financial Volatility: Stochastic Volatility and Lévy Based Models. Cambridge: Cambridge University Press.

Bättig, Robert. 1999. Completeness of securities market models-an operator point of view. Annals of Applied Probability 9: 529-66. [CrossRef]

Bättig, Robert, and Robert A. Jarrow. 1999. The second fundamental theorem of asset pricing: A new approach. The Review of Financial Studies 12: 1219-35. [CrossRef]

Bäuerle, Nicole, and Ulric Rieder. 2004. Portfolio optimization with Markov-modulated stock prices and interest rates. IEEE Transactions on Automatic Control 49: 442-47. [CrossRef]

Bäuerle, Nicole, and Ulric Riede. 2007. Portfolio optimization with jumps and unobservable intensity process. Mathematical Finance 17: 205-24. [CrossRef]

Björk, Tomas, and Bertil Näslund. 1998. Diversified portfolios in continuous time. European Financial Review 1: 361-87. [CrossRef]

Black, Fischer, Michael C. Jensen, and Myron Scholes. 1972. Some Empirical Tests, Studies in the Theory of Capital Markets. In Studies in the Theory of Capital Markets. Edited by M. Jensen. New York: Praeger, pp. 79-121.

Boel, René, and Michael Kohlmann. 1980. Semimartingale models of stochastic optimal control, with applications to double Martingales. SIAM Journal on Control and Optimization 18: 511-33. [CrossRef]

Buffington, John, and Robert J. Elliott. 2002. American options with regime switching. International Journal of Theoretical and Applied Finance 5: 497-514. [CrossRef]

Busch, Michael, Ralf Korn, and Frank Thomas Seifried. 2013. Optimal consumption and investment for a large investor: An intensity-based control framework. Mathematical Finance 23: 687-717. [CrossRef]

Çinlar, Erhan. 1972a. Markov additive processes: I. Zeitschrift Wahrscheinlichkeitstheorie und verwandte Gebiete 24: 85-93. [CrossRef]

Çinlar, Erhan. 1972b. Markov additive processes: II. Zeitschrift Wahrscheinlichkeitstheorie und verwandte Gebiete 24: 95-121. [CrossRef]

Corcuera, José Manuel, Joao Guerra, David Nualart, and Wim Schoutens. 2006. Optimal investment in a Lévy market. Applied Mathematics and Optimization 53: 279-309. [CrossRef]

Corcuera, José Manuel, David Nualart, and Wim Schoutens. 2005. Completion of a Lévy market by power-jump assets. Finance and Stochastics 9: 109-27. [CrossRef]

Cuchiero, Christa, Irene Klein, and Josef Teichmann. 2016. A new perspective on the fundamental theorem of asset pricing for large financial markets. Theory of Probability and its Applications 60: 561-79. [CrossRef]

Di Masi, Giovanni Battista, Yuri M. Kabanov, and Wolfgang J. Runggaldier. 1994. Mean variance hedging of options on stocks with Markov volatility. Theory of Probability and Applications 39: 173-81. [CrossRef]

Elliott, Rogemar J., Lakhdar Aggoun, and John B. Moore. 1994. Hidden Markov Models: Estimation and Control. Berlin: Springer.

Elliott, Rogemar J., Leunglung Chan, and Tak Kuen Siu. 2005. Option pricing and esscher transform under regime switching. Annals of Finance 1: 423-32. [CrossRef]

Elliott, Rogemar J., William C. Hunter, and Barbara M. Jamieson. 2001. Financial signal processing: A self calibrating model. International Journal of Theoretical and Applied Finance 4: 567-84. [CrossRef]

Elliott, Rogemar J., W. P. Malcolm, and Allanus H. Tsoi. 2003. Robust parameter estimation for asset price models with Markov modulated volatilities. Journal of Economic Dynamics and Control 27: 1391-409. [CrossRef]

Fechner, Włodzimierz. 2008. Some inequalities connected with the exponential function. Archivum Mathematicum (Brno) 44: 217-22.

Fleming, Wendell H., and Daniel Hernández-Hernández. 2003. An optimal consumption model with stochastic volatility. Finance and Stochastics 7: 245-62. [CrossRef]

Fontana, Claudio, Bernt Øksendal, and Agnès Sulem. 2015. Market viability and martingale measures under partial information. Methodology and Computing in Applied Probability 17: 15-39. [CrossRef]

Fouque, Jean-Pierre, Andrew Papanicolaou, and Ronnie Sircar. 2017a. Perturbation Analysis for Investment Portfolios Under Partial Information with Expert Opinions. SIAM Journal on Control and Optimization 55: 1534-66. [CrossRef] 
Fouque, Jean-Pierre, Ronnie Sircar, and T. Zariphopoulou. 2017b. Portfolio optimization and stochastic volatility asymptotics. Mathematical Finance 27: 704-45. [CrossRef]

Framstad, Nils Chr., Bernt Øksendal, and Agnes Sulem. 2004. Sufficient stochastic maximum principle for the optimal control of jump diffusions and applications to finance. Journal of Optimization Theory and Applications 121: 77-98. [CrossRef]

Fu, Jun, Jiaqin Wei, and Hailiang Yang. 2014. Portfolio optimization in a regime-switching market with derivatives. European Journal of Operational Research 233: 184-92. [CrossRef]

Gautam, Natarajan, Vidyadhar Kulkarni, Zbigniew Palmowski, and Tomasz Rolski. 1999. Bounds for fluid models driven by semi-Markov inputs. Probability in Engineering and Information Sciences 13: 429-75. [CrossRef]

Goldfeld, Stephen M., and Richard E. Quandt. 1973. A Markov model for switching regressions. Journal of Econometrics 1: 3-16. [CrossRef]

Goll, Thomas, and Jan Kallsen. 2000. Optimal portfolios for logarithmic utility. Stochastic Processes and their Applications 89: 31-48. [CrossRef]

Goll, Thomas, and Jan Kallsen. 2003. A complete explicit solution to the log-optimal portfolio problem. The Annals of Applied Probability 13: 774-99.

Guo, Xin. 2001. Information and option pricings. Quantitative Finance 1: 38-44. [CrossRef]

Guo, Xin, Jianjun Miao, and Erwan Morellec. 2005. Irreversible investments with regime shifts. Journal of Economic Theory 122: 37-59. [CrossRef]

Gut, Allan. 2005. Probability: A Graduate Course. New York: Springer.

Hamilton, James D. 1989. A new approach to the economics analysis of non-stationary time series. Econometrica 57: 357-84. [CrossRef]

Heston, Steven. 1993. A closed-form solution for options with stochastic volatility with applications to bond and currency options. Review of Financial Studies 6: 327-43. [CrossRef]

Jacod, Jean, and Philip Protter. 2004. Probability Essentials, 2nd ed. Berlin and Heidelberg: Springer.

Kabanov, Yuri, and Dmitry Kramkov. 1994. Large financial markets: Asymptotic arbitrage and contiguity. Probability Theory and Its Applications 39: 222-29. [CrossRef]

Kabanov, Yuri, and Dmitry Kramkov. 1998. Asymptotic arbitrage in large financial markets. Finance and Stochastics 2: 143-72. [CrossRef]

Karatzas, Ioannis, John P. Lehoczky, Steven E. Shreve, and Gan-Lin Xu. 1991. Martingale and duality methods for utility maximization in an incomplete market. SIAM Journal on Control and Optimization 29: 702-30. [CrossRef]

Karatzas, Ioannis, and Steven E. Shreve. 1998. Brownian Motion and Stochastic Calculus. Berlin, Heidelberg and New York: Springer.

Korn, Ralf, Yaroslav Melnyk, and Frank Thomas Seifried. 2017. Stochastic impulse control with regime-switching dynamics. European Journal of Operational Research 260: 1024-42. [CrossRef]

Korn, Ralf, and H. Kraft. 2001. A stochastic control approach to portfolio problems with stochastic interest rates. SIAM Journal on Control and Optimization 40: 1250-69. [CrossRef]

Kramkov, Dmitry, and Walter Schachermayer. 1999. The asymptotic elasticity of utility functions and optimal investment in incomplete markets. Annals of Applied Probability 9: 904-50. [CrossRef]

Kreps, David M. 1981. Arbitrage and equilibrium in economies with infinitely many commodities. Journal of Mathematical Economics 8: 15-35. [CrossRef]

Kushner, Harold J., and Paul G. Dupuis. 1992. Numerical Methods for Stochastic Control Problems in Continuous Time. New York: Springer.

Liao, Ming. 2004. Lévy Processes in Lie Groups. Cambridge Tracts in Mathematics. Cambridge: Cambridge University Press, vol. 162.

Liu, Ruihua. 2014. Optimal investment and consumption with proportional transaction costs in regime-switching model. Journal of Optimization Theory and Applications 163: 614-41. [CrossRef]

Markowitz, Harry M. 1952. Portfolio selection. The Journal of Finance 7: 77-91.

Merton, Robert C. 1971. Optimal consumption and portfolio rules in a continuous time model. Journal of Economic Theory 3: 373-413. [CrossRef]

Merton, Robert C. 1976. Option pricing when underlying stock returns are discontinuous. Journal of Financial Economics 3: 125-44. [CrossRef] 
Merton, Robert C. 1980. On estimating the expected return on the market. Journal of Financial Economics 8: $323-61$. [CrossRef]

Mokkhavesa, Sutee, and Colin Atkinson. 2002. Perturbation solution of optimal portfolio theory with transaction costs for any utility function. IMA Journal of Management Mathematics 13: 131-51. [CrossRef]

Nagai, Hideo, and Wolfgang J. Runggaldier. 2008. PDE Approach to Utility Maximization for Market Models with Hidden Markov Factors. In Seminar on Stochastic Analysis, Random Fields and Applications V, Progress in Probability. Edited by R. C. Dalang, M. Dozzi and F. Russo. Basel: Birkhuser Verlag, vol. 59, pp. 493-506.

Naik, Vasanttilak. 1993. Option valuation and hedging strategies with jumps in the volatility of asset returns. Journal of Finance 48: 1969-84. [CrossRef]

Niu, Liqun. 2008. Some stability results of optimal investment in a simple Lévy market. Insurance Mathematics Economics 42: 445-52. [CrossRef]

Øksendal, Bernt, and Agnès Sulem. 2004. Applied Stochastic Control of Jump Diffusions. Berlin and Heidelberg: Springer.

Pacheco, António, Loon Ching Tang, and Narahari U. Prabhu. 2009. Markov-Modulated Processes and Semigenerative Phenomena. Hackensack: World Scientific.

Palmowski, Zbigniew, Łukasz Stettner, and Anna Sulima. 2018. A note on chaotic and predictable representations for Itô-Markov additive processes. Stochastic Analysis and Applications 36: 622-38. [CrossRef]

Pham, Huyên Pham, and Marie-Claire Quenez. 2001. Optimal portfolio in partially observed stochastic volatility models. The Annals of Applied Probability 11: 210-38. [CrossRef]

Prabhu, Narahari U. 1998. Stochastic Storage Processes: Queues, Insurance Risk, Dams, and Data Communication, 2nd ed. Berlin and Heidelberg:Springer.

Protter, Philip E. 2005. Stochastic Integration and Differential Equations: A New Approach, 2nd ed. Berlin: Springer.

Resnick, Sidney I. 2007. Heavy-Tail Phenomena. Probabilistic and Statistical Modeling. New York: Springer.

Rieder, Ulrich, and Nicole Bäuerle. 2005. Portfolio optimization with unobservable markov-modulated drift process. Journal of Applied Probability 42: 362-78. [CrossRef]

Sass, Jörn, and Ulrich G. Haussmann. 2004. Optimizing the terminal wealth under partial information: The drift process as a continuous time markov chain. Finance and Stochastics 8: 553-77. [CrossRef]

Schoutens, Wim. 1999. Stochastic Processes and Orthogonal Polynomials. Lecture Notes in Statistics. New York: Springer, vol. 146.

Shen, Yang, and Tak Kuen Siu. 2012. Asset allocation under stochastic interest rate with regime switching. Economic Modelling 29: 1126-36. [CrossRef]

Sotomayor, Luz R., and Abel Cadenillas. 2013. Stochastic impulse control with regime switching for the optimal dividend policy when there are business cycles, taxes and fixed costs. Stochastics: An International Journal of Probability and Stochastic Processes 85: 707-22. [CrossRef]

Stockbridge, Richard. 2002. Portfolio optimization in markets having stochastic rates. In Lecture Notes in Control and Information Sciences: Stochastic Theory and Control. Edited by B. Pasik-Duncan. Berlin: Springer, vol. 280, pp. 447-58.

Teplá, Lucie. 2000. Optimal portfolio policies with borrowing and shortsale constraints. Journal of Economic Dynamics and Control 24: 1623-39. [CrossRef]

Tong, Howell. 1978. Threshold Models in Non-linear Time Series Analysis. Berlin: Springer.

Tu, Jun. 2010. Is regime switching in stock returns important in asset allocations? Management Science 56: 1198-215. [CrossRef]

Yin, George, and Xun Yu Zhou. 2004. Markowitz's mean-variance portfolio selection with regime switching: From discrete-time models to their continuous-time limits. IEEE Transactions on Automatic Control 49: 349-60. [CrossRef]

Zariphopoulou, Thaleia. 1992. Investment-consumption models with transaction fees and markov-chain parameters. SIAM Journal on Control and Optimization 30: 613-36. [CrossRef]

Zhang, Xin, Robert J. Elliott, Tak Kuen Siu, and Junyi Guo. 2012. Markovian regime-switching market completion using additional Markov jump assets. IMA Journal of Management Mathematics 23: 283-305. [CrossRef]

Zhang, Qing. 2001. Stock trading: An optimal selling rule. SIAM Journal on Control and Optimization 40: 64-87. [CrossRef]

Zhang, Xin, Tak Kuen Siu, and Qingbin Meng. 2010. Porfolio selection in the enlarged Markovian regime-switching market. SIAM Journal on Control and Optimization 48: 3368-88. [CrossRef] 
Zhou, Xun Yu, and George Yin. 2003. Markowitz's Mean-Variance Portfolio Selection with Regime Switching: A Continuous Time Model. SIAM Journal on Control and Optimization 42: 1466-82. [CrossRef]

Zhang, Qing, and George Yin. 2004. Nearly-optimal asset allocation in hybrid stock investment models. Journal of Optimization Theory and Applications 121: 197-222. [CrossRef]

(c) ( (C) 2019 by the authors. Licensee MDPI, Basel, Switzerland. This article is an open access article distributed under the terms and conditions of the Creative Commons Attribution (CC BY) license (http:/ / creativecommons.org/licenses/by/4.0/). 